\title{
MIDGAP DEFECT MODES IN DIELECTRIC AND ACOUSTIC MEDIA*
}

\author{
ALEXANDER FIGOTIN ${ }^{\dagger}$ AND ABEL KLEIN ${ }^{\ddagger}$
}

\begin{abstract}
We consider three dimensional lossless periodic dielectric (photonic crystals) and acoustic media having a gap in the spectrum. If such a periodic medium is perturbed by a strong enough defect, defect eigenmodes arise, localized exponentially around the defect, with the corresponding eigenvalues in the gap. We use a modified Birman-Schwinger method to derive equations for these eigenmodes and corresponding eigenvalues in the gap, in terms of the spectral attributes of an auxiliary Hilbert-Schmidt operator. We prove that in three dimensions, under some natural conditions on the periodic background, the number of eigenvalues generated in a gap of the periodic operator is finite, and give an estimate on the number of these midgap eigenvalues. In particular, we show that if the defect is weak there are no midgap eigenvalues.
\end{abstract}

Key words. photonic crystal, photonic bandgap, periodic acoustic medium, periodic dielectric medium, midgap states, defect modes, localization of light

AMS subject classifications. 78A40, 76N99, 35P99, 35Q60, 35Q35

PII. S0036139997320536

1. Introduction. Localization of classical waves, acoustic and electromagnetic, has received much attention in recent years (e.g., [An, J1, J2, DE, Sc, VP, M, HJ, JMW] and references therein). This phenomenon arises from coherent multiple scattering and interference and occurs when the scale of the coherent multiple scattering reduces to the wavelength itself. Numerous potential applications (e.g., [DE, J2, VP, HJ]), for instance, the optical transistor, and the fundamental significance of the localization of classical waves motivate the interest in this phenomenon.

In this article we continue our study of localization phenomena due to a single defect in a periodic medium. (A periodic dielectic medium is called a photonic crystal (e.g., [JMW])). In a previous article [FK4], we showed that defects do not change the essential spectrum of the associated nonnegative operators. Thus the effect of the defect inside a spectral gap of the periodic medium can only be the creation of isolated eigenvalues of finite multiplicity, with the corresponding eigenmodes decaying exponentially away from the defect. These are usually called midgap eigenvalues and defect eigenmodes. We also gave a constructive and simple description of defects in acoustic and dielectric media, including a simple condition on the parameters of the

${ }^{*}$ Received by the editors April 24, 1997; accepted for publication (in revised form) August 18, 1997; published electronically August 3, 1998. This research was supported by the Air Force Office of Scientific Research, Air Force Materials Command, USAF, under grant F49620-97-0019, and by the Division of Mathematical Sciences of the National Science Foundation, under grant DMS-9500720. The U.S. Government is authorized to reproduce and distribute reprints for governmental purposes notwithstanding any copyright notation thereon. The views and conclusions contained herein are those of the authors and should not be interpreted as necessarily representing the official policies or endorsements, either expressed or implied, of the Air Force Office of Scientific Research, the National Science Foundation, or the US Government.

http://www.siam.org/journals/siap/58-6/32053.html

${ }^{\dagger}$ Department of Mathematics, University of North Carolina at Charlotte, Charlotte, NC 28223 (figotin@uncc.edu). The research of this author was supported by the U.S. Air Force grant F4962094-1-0172.

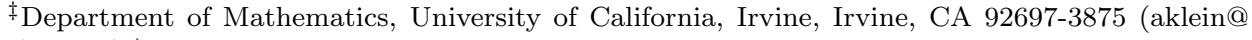
math.uci.edu). 
medium and of the defect, which ensures the rise of an defect eigenmode with the corresponding eigenvalue in a specified subinterval of the given gap of the periodic medium.

We have previously given rigorous proofs of Anderson localization due to a random array of defects, both in the lattice [FK1, FK2] and in the continuum [FK3, FK5], including the true three-dimensional continuum case.

We consider acoustic and electromagnetic waves in a lossless, inhomogeneous medium; they are described by the formally self-adjoint operators

$$
A=A(\varepsilon)=-\nabla \cdot \frac{1}{\varepsilon(x)} \nabla \text { on } L^{2}\left(\mathbb{R}^{d}\right),
$$

and

$$
\mathbf{M}=\mathbf{M}(\varepsilon)=\nabla^{\times} \frac{1}{\varepsilon(x)} \nabla^{\times} \text {on } \mathbb{S},
$$

where $\mathbb{S}$, the space of solenoidal fields, is the closure in $L^{2}\left(\mathbb{R}^{3} ; \mathbb{C}^{3}\right)$ of the linear subset $\left\{\Psi \in C_{0}^{1}\left(\mathbb{R}^{3} ; \mathbb{C}^{3}\right) ; \nabla \cdot \Psi=0\right\}$. We use the notation

$$
\nabla^{\times} \Psi=\nabla \times \Psi=\operatorname{curl} \Psi ; \quad \nabla \cdot \Psi=\operatorname{div} \Psi .
$$

We also set

$$
M=M(\varepsilon)=\nabla^{\times} \frac{1}{\varepsilon(x)} \nabla^{\times} \text {on } L^{2}\left(\mathbb{R}^{3} ; \mathbb{C}^{3}\right) .
$$

The function $\varepsilon(x)$ describes the medium; it is the position dependent mass density for acoustic waves and the position dependent dielectric constant for electromagnetic waves. We deliberately pick the same notation $\varepsilon(x)$ for the coefficients of the above operators, even so they have different physical meaning, in order to emphasize their similarity and describe uniformly their common spectral properties.

We always assume that $\varepsilon(x)$ is a measurable real-valued function satisfying

$$
0<\varepsilon_{-} \leq \varepsilon(x) \leq \varepsilon_{+}<\infty \text { a.e. for some constants } \varepsilon_{-} \text {and } \varepsilon_{+} .
$$

Localized acoustic or electromagnetic waves are finite energy solutions of the acoustic or Maxwell equations with the property that almost all of the wave's energy remains in a fixed bounded region of space at all times. They can be constructed from exponentially localized eigenmodes (e.g., defect eigenmodes) of the acoustic operator $A$ or the Maxwell operator M. (See the discussion in [FK3, FK5].)

A defect is a perturbation of a given medium in a finite domain. Defects in the medium can generate eigenmodes of $A$ or $\mathbf{M}$, with corresponding eigenvalues in spectral gaps of the underlying unperturbed medium. This phenomenon is analogous to the rise of bound states for electrons described by Schrödinger operators, due to defects such as a well potential satisfying some simple conditions on its width and depth (e.g., [S]). To employ a mechanism for localization of classical waves similar to the one for electronic localization, we started in [FK4] with a medium described by a coefficient $\varepsilon_{0}(x)$, such that the corresponding acoustic or Maxwell operator has a gap inside its spectrum with the edges depending on the medium, i.e., on the coefficient $\varepsilon_{0}(x)$. We proved that if such medium is perturbed locally by a defect (see Fig. 1), the corresponding acoustic or Maxwell operator can exhibit exponentially localized 


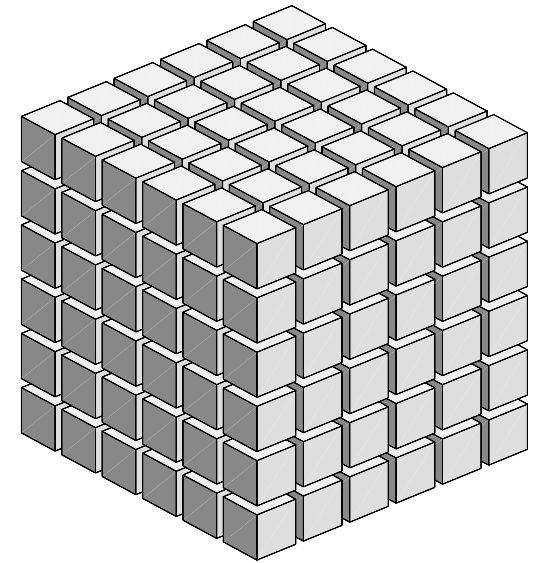

(a)

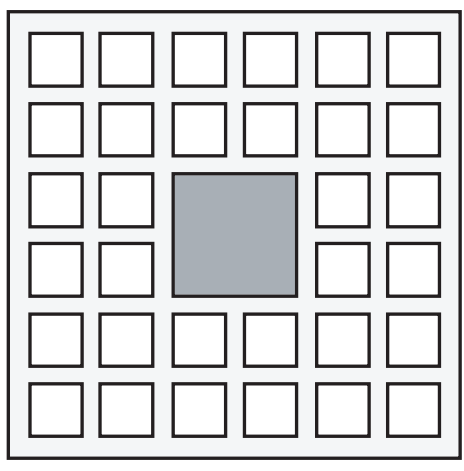

(b)

FIG. 1. A slab of a photonic crystal (a) with an interior defect of higher dielctric constant. The defect is shown on the crossection of the slab (b) as a darker square.

eigenmodes with corresponding eigenvalues in the interior of the gaps, the defect eigenmodes.

The most natural way to obtain media with gaps in the spectrum is to consider periodic media, i.e., media described by periodic $\varepsilon_{0}(x)$. In this case, the spectra of the corresponding operators $A_{0}$ and $M_{0}$, according to Floquet-Bloch theory, have bandgap structure and can have gaps. The existence of gaps for some periodic dielectric and acoustic media was proved in [FKu1, FKu2].

We showed in [FK4] that defects satisfying simple conditions generate defect eigenmodes with corresponding eigenvalues in the gaps, and that the interiors of the gaps contain no points of accumulation of those defect eigenvalues. In this paper, we use a modified Birman-Schwinger method to derive equations for these defect eigenmodes and corresponding midgap eigenvalues, in terms of the spectral attributes of an auxiliary Hilbert-Schmidt operator. We prove that in three-dimensions, under some natural conditions on the periodic background described by $\varepsilon_{0}(x)$, the number of eigenvalues generated in a gap of the periodic operator is finite, and give an estimate on the number of these midgap eigenvalues. In particular, we show that if the defect is not strong enough no eigenvalues will be generated in the gap, as in the case of three dimensional Schrödinger operators $-\Delta+V$, with $V$ a well potential, where bound states are not created below the continuous spectrum if the well is shallow (e.g., $[\mathrm{S}]$ ).

2. Statement of results. $A$ and $M$ are defined as the nonnegative self-adjoint operators on $L^{2}\left(\mathbb{R}^{d}\right)$ and $L^{2}\left(\mathbb{R}^{3} ; \mathbb{C}^{3}\right)$. They are uniquely defined by the closure of the nonnegative densely defined quadratic forms

$$
\mathcal{A}(\psi)=\left\langle\nabla \psi, \frac{1}{\varepsilon(x)} \nabla \psi\right\rangle \equiv \sum_{j=1}^{d}\left\langle\partial_{j} \psi, \frac{1}{\varepsilon(x)} \partial_{j} \psi\right\rangle, \text { with } \psi \in C_{0}^{1}\left(\mathbb{R}^{d}\right),
$$

and

$$
\mathcal{M}(\Psi)=\left\langle\nabla \times \Psi, \frac{1}{\varepsilon(x)} \nabla \times \Psi\right\rangle \text {, with } \Psi \in C_{0}^{1}\left(\mathbb{R}^{3} ; \mathbb{C}^{3}\right)
$$


We recall Weyl's decomposition (see $[\mathrm{BS}]), L^{2}\left(\mathbb{R}^{3} ; \mathbb{C}^{3}\right)=\mathbb{S} \oplus \mathbb{G}$, where $\mathbb{G}$, the space of potential fields, is the closure in $L^{2}\left(\mathbb{R}^{3} ; \mathbb{C}^{3}\right)$ of the linear subset $\{\Psi \in$ $C_{0}^{1}\left(\mathbb{R}^{3} ; \mathbb{C}^{3}\right) ; \quad \Psi=\nabla \varphi$ with $\left.\varphi \in C_{0}^{1}\left(\mathbb{R}^{3}\right)\right\}$. The spaces $\mathbb{S}$ and $\mathbb{G}$ are invariant under $M$, with $\mathbb{G} \subset \mathcal{D}(M)$ and $\left.M\right|_{\mathbb{G}}=0$. We define $\mathbf{M}$ as the restriction of $M$ to $\mathbb{S}$, i.e., $\mathcal{D}(\mathbf{M})=\mathcal{D}(M) \cap \mathbb{S}$ and $\mathbf{M}=\left.M\right|_{\mathcal{D}(M) \cap \mathbb{S}}$. Thus,

$$
\mathbf{M}=P_{\mathbb{S}} M I_{\mathbb{S}}=M I_{\mathbb{S}}
$$

with $P_{\mathbb{S}}$ the orthogonal projection onto $\mathbb{S}$ and $I_{\mathbb{S}}: \mathbb{S} \rightarrow L^{2}\left(\mathbb{R}^{3} ; \mathbb{C}^{3}\right)$, the restriction of the identity map. Note $M=\mathbf{M} \oplus 0_{\mathbb{G}}$, so we can work with $M$ to answer questions about the spectrum of $\mathbf{M}$.

In this article we discuss results common to both acoustic and Maxwell operators. Since most of the discussion will apply to both cases, where it simplifies the discussion we will use $W$ to denote either $A$ or $\mathbf{M}$, and $\widetilde{W}$ to denote either $A$ or $M$. We will also write $R(z)=(W-z I)^{-1}$ and $\widetilde{R}(z)=(\widetilde{W}-z I)^{-1}$ for the resolvents. In addition, we will use $\breve{R}(z)$ for either $(A-z I)^{-1}$ or $(\mathbf{M}-z I)^{-1} \oplus 0_{\mathbb{G}}$. Note $d=3$ if $W=\mathbf{M}$.

In this article the background medium will be described by a function $\varepsilon_{0}(x)$ as in (4), which will be taken to be periodic with period $q: \varepsilon_{0}(x)=\varepsilon_{0}(x+q m)$ for all $x \in \mathbb{R}^{d}$ and $m \in \mathbb{Z}^{d}$. We take $q=1$ without loss of generality. The corresponding operators will be denoted by $A_{0}, \mathbf{M}_{0}, M_{0}, W_{0}, \widetilde{W}_{0}, R_{0}(z), \widetilde{R}_{0}(z), \breve{R}_{0}(z)$. An interval $(a, b)$ will be called a gap in the spectrum of $W_{0}$, if $a, b \in \sigma\left(W_{0}\right)$ with $a<b$, and $\sigma\left(W_{0}\right) \bigcap(a, b)=\emptyset$.

We will say that the medium described by $\varepsilon(x)$ (as in (4)) was obtained from the background medium by the insertion of a defect, if $\varepsilon(x)$ and $\varepsilon_{0}(x)$ differ only in a bounded domain, i.e., $\varepsilon(x)-\varepsilon_{0}(x)$ has compact support; we will then say that $\varepsilon(x)$ and $\varepsilon_{0}(x)$ differ by a defect. In this case the following results are proved in [FK4].

Stability of essential spectrum: $W$ and $W_{0}$ have the same essential spectrum, i.e., $\sigma_{\text {ess }}(W)=\sigma_{\text {ess }}\left(W_{0}\right)$. If $(a, b)$ is a gap in the spectrum of $W_{0}$, the spectrum of $W$ in $(a, b)$ consists at most of isolated eigenvalues with finite multiplicity, with the corresponding eigenmodes decaying exponentially fast, with a rate depending on the distance from the eigenvalue to the edges of the gap.

Creation of defect eigenmodes: Let $(a, b)$ be a gap in the spectrum of $W_{0}$, select $\mu \in(a, b)$, and pick $0<\gamma<1$ such that the interval $[\mu(1-\gamma), \mu(1+\gamma)]$ is contained in the gap, i.e., $[\mu(1-\gamma), \mu(1+\gamma)] \subset(a, b)$. If $\varepsilon(x) \equiv \epsilon$ in a cube of side $\ell$ with

$$
\ell^{2} \epsilon>\frac{24}{\mu \gamma^{2}}\left(1+\sqrt{1+\frac{d(5+2 d)}{8} \gamma^{2}}\right),
$$

the corresponding operator $W$ has at least one defect eigenmode, with corresponding eigenvalue inside the interval $[\mu(1-\gamma), \mu(1+\gamma)]$.

If either $\varepsilon(x) \geq \varepsilon_{0}(x)$ or $\varepsilon(x) \leq \varepsilon_{0}(x)$, we can say more about how the eigenvalues are distributed in the gap. We will call a defect positive if $\varepsilon(x) \leq \varepsilon_{0}(x)$, in which case we have $Y \equiv W-W_{0} \geq 0$. Similarly, a defect is negative if $\varepsilon(x) \geq \varepsilon_{0}(x)$, so $Y \leq 0$. We will also write $\widetilde{Y}=\widetilde{W}-\widetilde{W_{0}}$.

We would like to use the Birman-Schwinger method (e.g., [RS4, Kl, ADH]) to get more information about the eigenvalues a defect creates in a gap, but, unfortunately, $Y=W-W_{0}$ is not relatively compact with respect to $W_{0}$. The way out is to use the resolvents: we set $H=(W+I)^{-1}$ and $H_{0}=\left(W_{0}+I\right)^{-1}$, prove that $V=H-H_{0}$ is a Hilbert-Schmidt operator when $d \leq 3$ (see Proposition 5.1), and use the BirmanSchwinger method for $H=H_{0}+V$. (A different approach was used in [AADH, BL] to obtain asymptotic results for acoustic operators.) 
The Birman-Schwinger method gives equations for the defect eigenmodes and corresponding midgap eigenvalues in terms of the spectral attributes of an auxiliary Hilbert-Schmidt operator. Let $(a, b)$ be a gap in the spectrum of the operator $W_{0}$ and let us insert a negative defect, so $\varepsilon(x) \geq \varepsilon_{0}(x)$ and $V \geq 0$. Let us consider the eigenvalue problem for the operator $W$ in the gap

$$
W \psi=\mu \psi \text {, where } \mu \in(a, b) .
$$

This is clearly equivalent to the eigenvalue problem

$$
H \psi=(W+I)^{-1} \psi=(\mu+1)^{-1} \psi, \text { where } \mu \in(a, b) .
$$

On the other hand, the eigenvalue problem

$$
H \psi=H_{0} \psi+V \psi=\lambda \psi, \text { where } \lambda \notin \sigma\left(H_{0}\right)
$$

can be rewritten as

$$
\psi=-R_{0}(\lambda) V \psi, \text { where } R_{0}(\lambda)=\left(H_{0}-\lambda I\right)^{-1} .
$$

If we set

$$
\mathcal{R}(\lambda)=-\sqrt{V} R_{0}(\lambda) \sqrt{V},
$$

we obtain the eigenvalue problem

$$
\mathcal{R}(\lambda) \varphi=\varphi \text { with } \varphi=\sqrt{V} \psi,
$$

which is equivalent to the eigenvalue problem (11), as shown in Lemma 4.1. The Birman-Schwinger operator $\mathcal{R}(\lambda)$ is a self-adjoint Hilbert-Schmidt operator ( $V$ is Hilbert-Schmidt). The original eigenvalue problem (9) for $W$ can now be rewritten as follows:

$$
\mathcal{S}(\mu) \varphi=\varphi, \text { where } \varphi=\sqrt{V} \psi, \mu \in(a, b),
$$

and $\mathcal{S}(\mu)$ is the self-adjoint Hilbert-Schmidt operator given by

$$
\mathcal{S}(\mu)=\mathcal{R}\left((\mu+1)^{-1}\right)=(\mu+1) \sqrt{V} \frac{W_{0}+I}{W_{0}-\mu I} \sqrt{V} .
$$

In the case of a positive defect, so $\varepsilon(x) \leq \varepsilon_{0}(x)$ and $V \leq 0$, the analogue of the equations (15) and (16) takes the form

$$
\mathcal{S}(\mu) \varphi=-\varphi, \text { where } \varphi=\sqrt{-V} \psi, \mu \in(a, b),
$$

with

$$
\mathcal{S}(\mu)=(\mu+1) \sqrt{-V} \frac{W_{0}+I}{W_{0}-\mu I} \sqrt{-V} .
$$

Observe now that since $\left(W_{0}-\mu I\right)^{-1}$ is a monotonically increasing, norm-continuous operator function of $\mu \in(a, b)$, the operator $\mathcal{S}(\mu)$ is also a monotonically increasing, norm-continuous function of $\mu \in(a, b)$ for both negative and positive defects. Since 


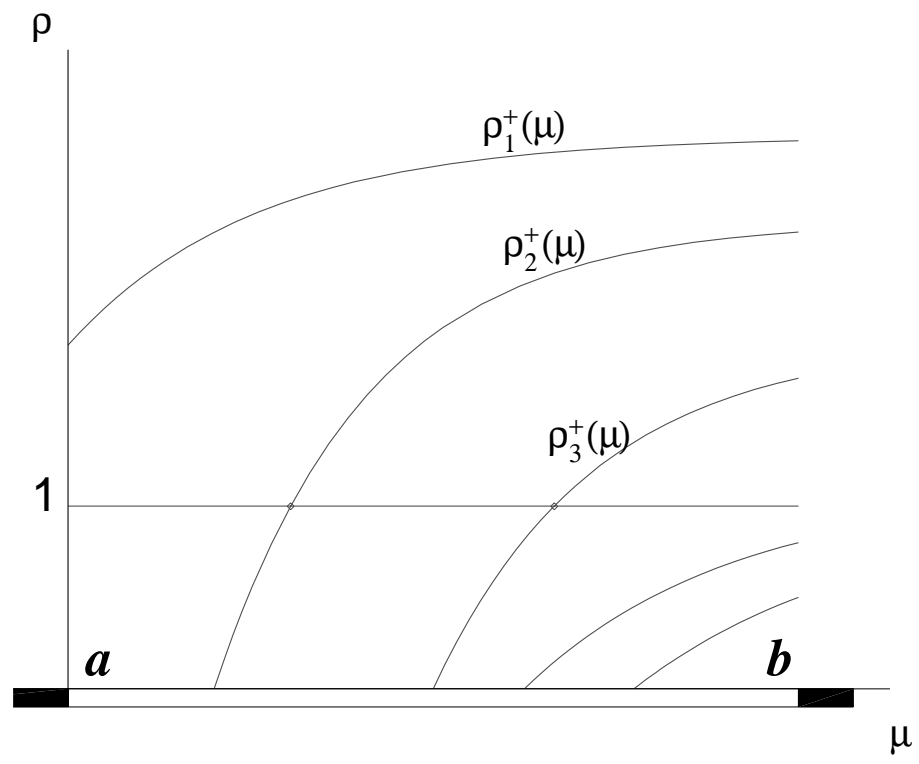

FIG. 2. The equations for the eigenvalues for the defect eigenmodes take the form $\rho_{n}^{+}(\mu)=1$ where the functions $\rho_{n}^{+}(\mu)$ are the eigenvalues of an auxiliary compact operator depending on the spectral parameter $\mu$.

$\mathcal{S}(\mu)$ is a self-adjoint Hilbert-Schmidt operator, its spectrum consists of eigenvalues of finite multiplicity with 0 being the only possible point of accumulation. Let $\rho_{1}^{+}(\mu) \geq \rho_{2}^{+}(\mu) \geq \cdots \geq 0$ and $\rho_{1}^{-}(\mu) \leq \rho_{2}^{-}(\mu) \leq \ldots \leq 0$ be the sequences of respectively, the positive and negative eigenvalues of the operator $\mathcal{S}(\mu)$, repeated according to their multiplicity. If we have a finite number of either positive or negative eigenvalues, we complete the sequence by assigning the value 0 . The functions $\rho_{n}^{ \pm}(\mu)$ are monotonically increasing and continuous in $\mu \in(a, b)$.

ThEOREM 2.1. Suppose $d \leq 3$. Let $\varepsilon_{0}(x)$ be a periodic function satisfying (4), such that the interval $(a, b)$ is a gap in the spectrum of $W_{0}$. Let $\varepsilon(x)$ be obtained from $\varepsilon_{0}(x)$ by the insertion of a defect. Then:

(i) If the defect is negative, the only possible point of accumulation of $\sigma(W) \cap$ $(a, b)$ is $b$. In this case the eigenvalues $\mu_{1} \leq \mu_{2} \leq \cdots$ of the operator $W$ in the gap $(a, b)$ coincide with the set of the solutions of the equations (see Fig. 2)

$$
\rho_{n}^{+}(\mu)=1, \quad n=1,2, \ldots,
$$

where $\rho_{n}^{+}(\mu)$ are the positive eigenvalues of the operator $\mathcal{S}(\mu)$ defined by (16). Moreover, if $\varphi_{i}$ is an eigenmode of the operator $\mathcal{S}\left(\mu_{i}\right)$ with eigenvalue $\rho_{n_{i}}^{+}\left(\mu_{i}\right)=1$, then

$$
\psi_{i}=\frac{W_{0}+I}{W_{0}-\mu_{i} I} \sqrt{V} \varphi_{i}
$$

is an exponentially localized eigenmode of the operator $W$ with eigenvalue $\mu_{i}$.

(ii) If the defect is positive, the only possible point of accumulation of $\sigma(W) \cap$ $(a, b)$ is $a$. In this case the eigenvalues $\mu_{1} \geq \mu_{2} \geq \cdots$ of the operator $W$ in the gap $(a, b)$ coincide with the set of the solutions of the equations

$$
\rho_{n}^{-}(\mu)=-1, \quad n=1,2, \ldots,
$$


where $\rho_{n}^{-}(\mu)$ are the negative eigenvalues of the operator $\mathcal{S}(\mu)$ defined by (18). Moreover, if $\varphi_{i}$ is an eigenmode of the operator $\mathcal{S}\left(\mu_{i}\right)$ with eigenvalue $\rho_{n_{i}}^{-}\left(\mu_{i}\right)=-1$, then

$$
\psi_{i}=-\frac{W_{0}+I}{W_{0}-\mu_{i} I} \sqrt{-V} \varphi_{i}
$$

is an exponentially localized eigenmode of the operator $W$ with eigenvalue $\mu_{i}$.

Note that for defects as in (8) with $\mu=\frac{a+b}{2}$ and $\gamma=\frac{b-a}{a+b}$, defect eigenmodes and midgap eigenvalues always exist (see [FK4, Theorem 2 and Remark 3] for more details), so in this case we can guarantee the existence of a solution for some of equations (19) and (21).

Theorem 2.1 follows from Proposition 5.1 and Theorem 4.4. It reduces the search for defect eigenmodes and midgap eigenvalues of the perturbed operator $W$ to the investigation of the spectral attributes of the relevant Hilbert-Schmidt operator $\mathcal{S}(\mu)$. When it comes to numerical estimations, the reduction to the Hilbert-Schmidt operator $\mathcal{S}(\mu)$ is quite valuable, since clearly $\mathcal{S}(\mu)$ is more suitable for truncations than the original unbounded differential operator $W$ with generally nonsmooth coefficient $\varepsilon(x)$.

In this article, we estimate the number of eigenvalues a defect creates in a gap. To do so, we need a suitable regularity of the resolvent $\left(W_{0}-z I\right)^{-1}$ in the vicinity of the edges of the spectral gap $(a, b)$. When $d=3$ we expect edges to be typically regular. To start with, $\breve{R}_{0}(z) \chi_{0}$ is a Hilbert-Schmidt operator for any $z \notin \sigma\left(W_{0}\right)$ [FK3, Proposition 42], [FK5, Theorem 18]. Floquet theory [RS4, Ku, FK5] tells us that the spectrum of the periodic operator $W_{0}$ is the union of a countable number of bands; more precisely, there exist continuous functions $\left\{\mu_{n}(k)\right\}_{n=1,2, \ldots}$ on $\mathbb{R}^{d}$ (the dispersion relations), periodic with period $2 \pi$, with $\mu_{n}(k) \leq \mu_{n+1}(k)$, such that

$$
\sigma\left(W_{0}\right)=\bigcup_{n=1,2, \ldots} \mu_{n}(\widetilde{Q}), \quad \text { where } \widetilde{Q}=[-\pi, \pi)^{d} .
$$

It follows from (4) and the min-max principle that for any closed finite interval $I \subset \mathbb{R}$, we have $\mu_{n}(\widetilde{Q}) \cap I=\emptyset$ except for at most finitely many $n$ 's. Thus, if $(a, b)$ is a gap in the spectrum of $W_{0}$, and $\left\{\mu_{n}(k)\right\}_{n=1,2, \ldots}$ are the corresponding dispersion relations, there must exist $n_{0} \in \mathbb{N}$ such that $\mu_{n}(\widetilde{Q}) \subset[0, a]$ for all $n \leq n_{0}$ and $\mu_{n}(\widetilde{Q}) \subset[b, \infty)$ for all $n>n_{0}$. In addition, there exist a positive integer $n_{1} \leq n_{0}$ such that $a \notin \mu_{n}(\widetilde{Q})$ for either $n<n_{1}$ or $n>n_{0}$, and $a=\max \mu_{n}(\widetilde{Q})$ for $n_{1} \leq n \leq n_{0}$.It follows that there exists a constant $c_{1}>0$ such that, if $n<n_{1}$ or $n>n_{0}$ we have $\left|a-\mu_{n}(k)\right| \geq c_{1}>0$ for all $k \in \widetilde{Q}$.

A natural regularity condition for the left edge $a$ is a nondegeneracy condition: for each $n_{1} \leq n \leq n_{0}$ we have $a=\mu_{n}(k)$ for only finitely many $k \in \widetilde{Q}$, say $k_{n, 1}, \ldots, k_{n, s_{n}}$, and for any $i=1, \ldots, s_{n}$ we have

$$
a-\mu_{n}(k) \geq c_{i}\left|k-k_{n, i}\right|^{2} \text { for }\left|k-k_{n, i}\right| \text { small, with } c_{i}>0,
$$

(with a similar definition for the right edge $b$ ).

This nondegeneracy condition is a common assumption in the physical literature (e.g., [HJ, Equation (4.53)]). It was also used in the study of Lipschitz tails in spectral gaps of periodic Schrödinger operators $[\mathrm{Kp}]$.

Verification of the regularity of a gap edge is not a simple matter even for Schrödinger operators. Below we give two rather natural definitions of regularity 
of a gap edge, sufficient for our purposes. Conditions under which one can guarantee the desired regularity is a subject for a separate investigation.

Definition 2.2. Let $(a, b)$ be a gap in the spectrum of $W_{0}$, let $\left\{\mu_{n}\right\}_{n=1,2, \ldots}$ be the corresponding dispersion relations, and let $c$ denote either the left edge a or the right edge $b$. We say that the edge $c$ is strongly regular if

$$
\int_{\widetilde{Q}} \frac{d k}{\left|\mu_{n}(k)-c\right|}<\infty
$$

for all $n=1,2, \ldots$

Note that in three dimensions strong regularity of an edge is a an immediate consequence of the nondegeneracy condition.

For our purposes a weaker definition suffices.

DEFINITION 2.3. We say that the left edge a of the gap $(a, b)$ in the spectrum of the periodic operator $W_{0}$ is regular if

$$
\xi(a) \equiv \underset{\eta \downarrow 0}{\limsup \operatorname{Tr}}\left[\left(\chi_{0}\left|\breve{R}_{0}(a+\eta)\right| \chi_{0}\right)^{2}\right]<\infty,
$$

where $\chi_{0}$ denotes the characteristic function of the cube of side 1 (i.e., the period of $\left.\varepsilon_{0}(x)\right)$ centered at the origin. Similarly, the right edge $b$ is regular if

$$
\xi(b) \equiv \limsup _{\eta \downarrow 0} \operatorname{Tr}\left[\left(\chi_{0}\left|\breve{R}_{0}(b-\eta)\right| \chi_{0}\right)^{2}\right]<\infty .
$$

To put the definition in perspective, note that for $z$ real and not in the spectrum of $W_{0}$, we always have $\operatorname{Tr}\left[\left(\chi_{0}\left|\breve{R}_{0}(z)\right| \chi_{0}\right)^{2}\right]<\infty$, since

$$
\begin{aligned}
\operatorname{Tr}\left[\left(\chi_{0}\left|\breve{R}_{0}(z)\right| \chi_{0}\right)^{2}\right] & =\left\|\chi_{0}\left|\breve{R}_{0}(z)\right| \chi_{0}\right\|_{2}^{2} \\
& \leq\left\|\left|\breve{R}_{0}(z)\right| \chi_{0}\right\|_{2}^{2}=\left\|\breve{R}_{0}(z) \chi_{0}\right\|_{2}^{2}<\infty
\end{aligned}
$$

as $\breve{R}_{0}(z) \chi_{0}$ is Hilbert-Schmidt by [FK3, Proposition 42], [FK5, Theorem 18], plus the resolvent identity. Furthermore, regularity follows from nondegeneracy; in fact it already follows from strong regularity.

THEOREM 2.4. When $d=3$, a strongly regular edge is always regular.

To formulate our main result we need a definition. For a given a self-adjoint operator $H$ and interval $(\alpha, \beta)$, we define

$$
N_{H}(\alpha, \beta)=\operatorname{Tr} \chi_{(\alpha, \beta)}(H) .
$$

Note $N_{H}(\alpha, \beta)$ is always a nonnegative integer unless it is infinite. If $H$ has discrete spectrum in $(\alpha, \beta), N_{H}(\alpha, \beta)$ gives the number of eigenvalues of $H$ in $(\alpha, \beta)$, counted according to their multiplicity.

It will be convenient to write

$$
\varepsilon(x)=\frac{\varepsilon_{0}(x)}{1+\theta(x)},
$$

where $\theta(x)$ is a bounded measurable function with compact support satisfying

$$
-1<\theta_{-} \leq \theta(x) \leq \theta_{+}<\infty \text { a.e. for some constants } \theta_{-} \text {and } \theta_{+} .
$$


In this case we will always take

$$
\varepsilon_{+}=\frac{\varepsilon_{0,+}}{1+\theta_{-}} \quad \text { and } \quad \varepsilon_{-}=\frac{\varepsilon_{0,-}}{1+\theta_{+}} .
$$

Note that a defect is positive if $\theta(x) \geq 0$, in which case we have $\theta_{-}=0$. Similarly, a defect is negative if $\theta(x) \leq 0$, so $\theta_{+}=0$.

Our main result is given in the following theorem.

TheOrem 2.5. Suppose $d=3$. Let $\varepsilon_{0}(x)$ be a periodic function satisfying (4), with the interval $(a, b)$ a gap in the spectrum of $W_{0}$. Let us insert a defect by taking $\varepsilon(x)$ be as in (30), where $\theta(x)$ satisfies (31) and vanishes outside a cube $\Lambda_{\ell}$ of side $\ell>0$. Letting $0<\delta \leq 1$, we have:

(i) Suppose the left edge $a$ is regular and the defect is positive, then

$$
N_{W}(a, b) \leq C_{a, \varepsilon_{0, \pm}, \theta_{+}, \delta} \theta_{+}^{2}(\ell+3)^{9+\delta} \xi(a)<\infty,
$$

with

$$
C_{a, \varepsilon_{0, \pm}, \theta_{+}, \delta}=\frac{C(a+1)^{2}}{\delta}\left[1+\varepsilon_{-}^{-(6+\delta)}\right]\left[1+6 \frac{\sqrt{\varepsilon_{0,+}}}{\varepsilon_{0,-}}\left(\sqrt{\varepsilon_{-}}+\frac{1}{\sqrt{\varepsilon_{-}}}\right)\right]^{2},
$$

where $C$ is some constant, independent of $\delta$ and of the other parameters, and $\varepsilon_{-}=$ $\frac{\varepsilon_{0,-}}{1+\theta_{+}}$.

(ii) Suppose the right edge $b$ is regular and the defect is negative, then

$$
N_{W}(a, b) \leq C_{b, \varepsilon_{0, \pm}, \theta_{-}, \delta} \theta_{-}^{2}(\ell+3)^{9+\delta} \xi(b)<\infty,
$$

with

$$
C_{b, \varepsilon_{0, \pm}, \theta_{-}, \delta}=\frac{C(b+1)^{2}}{\delta}\left[1+\varepsilon_{0,-}^{-(6+\delta)}\right]\left[1+6 \frac{\sqrt{\varepsilon_{+}}}{\varepsilon_{0,-}}\left(\sqrt{\varepsilon_{0,-}}+\frac{1}{\sqrt{\varepsilon_{0,-}}}\right)\right]^{2},
$$

where $C$ is some constant, independent $\delta$ and of the other parameters, and $\varepsilon_{+}=\frac{\varepsilon_{0,+}}{1+\theta_{-}}$.

Since the function $N_{W}(a, b)$ is integer valued, $N_{W}(a, b)<1$ implies $N_{W}(a, b)=0$. Thus we have the following immediate corollary, which tells us that there are no midgap eigenvalues if the defect is small.

Corollary 2.6. Suppose $d=3$. Let $\varepsilon_{0}(x)$ be a periodic function satisfying (4), with the interval $(a, b)$ a gap in the spectrum of $W_{0}$. Let us insert a defect by taking $\varepsilon(x)$ be as in (30), where $\theta(x)$ satisfies (31) and vanishes outside a cube $\Lambda_{\ell}$ of side $\ell>0$. Then:

(i) If the left edge $a$ is regular and the defect is positive, we must have $N_{W}(a, b)=$ 0 for $\theta_{+}>0$ small, how small depending only on $a, \varepsilon_{0, \pm}$ and $\ell$. In other words, there are no eigenvalues in the gap for weak defects.

(ii) If the right edge $b$ is regular and the defect is negative, we must have $N_{W}(a, b)=$ 0 for $\theta_{-}<0$ small, how small depending only on $b, \varepsilon_{0, \pm}$ and $\ell$, so there are no eigenvalues in the gap for weak defects.

For the reader's convenience we list some notations used throughout the paper:

- $W$ denotes either $A$ or $\mathbf{M}$, and $\widetilde{W}$ denotes either $A$ or $M$. The corresponding acoustic or dielectric medium is described by $\varepsilon(x)$.

- $R(z)=(W-z I)^{-1}, \widetilde{R}(z)=(\widetilde{W}-z I)^{-1}$.

- $\breve{R}(z)$ denotes either $(A-z I)^{-1}$ or $(\mathbf{M}-z I)^{-1} \oplus 0_{\mathbb{G}}$. 
- $A_{0}, \mathbf{M}_{0}, M_{0}, W_{0}, \widetilde{W}_{0}, R_{0}(z), \widetilde{R}_{0}(z), \breve{R}_{0}(z)$ correspond to the acoustic or dielectric medium described by $\varepsilon_{0}(x)$.

- $Y=W-W_{0}, \widetilde{Y}=\widetilde{W}-\widetilde{W}_{0}$.

- \#S is the number of elements in the set $S$.

- $\chi_{S}$ is the characteristic function of the set $S$.

- A bounded operator $T$, from a Hilbert space $\mathcal{H}_{1}$ to another Hilbert space $\mathcal{H}_{2}$, is of class $\mathcal{T}_{p}$, where $1 \leq p<\infty$, if $\|T\|_{p} \equiv\left[\operatorname{Tr}\left(T^{*} T\right)^{p / 2}\right]^{1 / p}<\infty$. In this case we will write $T \in \mathcal{T}_{p}$. Recall that $T$ is a Hilbert-Schmidt operator if and only if $T \in \mathcal{T}_{2}$.

3. Strongly regular edges. In this section we will prove Theorem 2.4. Let $Q=\left[-\frac{1}{2}, \frac{1}{2}\right)^{d}$ be the basic period cell, $\tilde{Q}=[-\pi, \pi)^{d}$ the dual basic cell. We define the Floquet transform (e.g., [FK5])

$$
\mathcal{F}: \quad L^{2}\left(\mathbb{R}^{d} ; \mathbb{C}^{\nu}\right) \rightarrow \int_{\tilde{Q}}^{\oplus} L^{2}\left(Q ; \mathbb{C}^{\nu}\right) d k \equiv L^{2}\left(\tilde{Q} ; L^{2}\left(Q ; \mathbb{C}^{\nu}\right)\right)
$$

by

$$
(\mathcal{F} \Psi)(k, x)=\widehat{\Psi}(k, x) \equiv\left(\frac{1}{2 \pi}\right)^{d / 2} \sum_{m \in \mathbb{Z}^{d}} \mathrm{e}^{i k \cdot(x-m)} \Psi(x-m), \quad x \in Q, k \in \tilde{Q}
$$

for $\Psi$ with compact support; it extends by continuity to a unitary operator. We will write $\widehat{B}=\mathcal{F} B \mathcal{F}^{*}$ for an operator $B$ on $L^{2}\left(\mathbb{R}^{d} ; \mathbb{C}^{\nu}\right)$. The periodic operator $\widetilde{W}_{0}$ is decomposable in this direct integral representation, more precisely,

$$
\widehat{\widehat{W}}_{0}=\mathcal{F} \widetilde{W}_{0} \mathcal{F}^{*}=\int_{\tilde{Q}}^{\oplus} \widetilde{W}_{0, Q}(k) d k,
$$

where for each $k \in \mathbb{R}^{d}$, we define $\widetilde{W}_{0, Q}(k)$ to be the nonnegative operator on $L^{2}\left(Q ; \mathbb{C}^{\nu}\right)$, given by either $-(\nabla-i k) \cdot \frac{1}{\varepsilon_{0}}(\nabla-i k)$ if $W=A$ or $(\nabla-i k)^{\times} \frac{1}{\varepsilon_{0}}(\nabla-i k)^{\times}$if $W=\mathbf{M}$, with periodic boundary condition; $\widetilde{W}_{0, Q}(k)$ is rigorously defined as a self-adjoint operator by the appropriate quadratic form. (As before $(\nabla-i k)^{\times}$denotes the operator $\left.(\nabla-i k)^{\times} \Phi=(\nabla-i k) \times \Phi.\right)$ In addition, the map

$$
k \in \mathbb{R}^{d} \longmapsto\left(\widetilde{W}_{0, Q}(k)+I\right)^{-1} \in \mathcal{L}\left(L^{2}\left(Q ; \mathbb{C}^{\nu}\right)\right)
$$

is operator norm continuous. (See [FK5, Subsection 3.3] for details.)

If $W=\mathbf{M}$, we define $\mathbb{S}_{Q}(k)$ to be the closure in $L^{2}\left(Q ; \mathbb{C}^{3}\right)$ of the linear space of periodic continuously differentiable functions $\Psi$ on $Q$ with $(\nabla-i k) \cdot \Psi=0$, and define $\mathbf{M}_{0, Q}(k)$ as the restriction of $M_{0, Q}(k)$ to $\mathbb{S}_{Q}(k)$. We have

$$
\mathcal{F} \mathbb{S}=\int_{\tilde{Q}}^{\oplus} \mathbb{S}_{Q}(k) d k, \quad \mathcal{F} \mathbf{M}_{0} \mathcal{F}^{*}=\int_{\tilde{Q}}^{\oplus} \mathbf{M}_{0, Q}(k) d k .
$$

Thus there is a direct integral representation for $W_{0}$

$$
\widehat{W_{0}}=\mathcal{F} W_{0} \mathcal{F}^{*}=\int_{\tilde{Q}}^{\oplus} W_{0, Q}(k) d k \quad \text { on } \int_{\tilde{Q}}^{\oplus} \mathcal{H}(k) d k,
$$

where each $W_{0, Q}(k)$ has compact resolvent [FK3, FK5]. Here $\mathcal{H}(k)$ denotes either $L^{2}(Q ; \mathbb{C})$ if $W=A$ or $\mathbb{S}_{Q}(k)$ if $W=\mathbf{M}$. 
It follows [RS4, Problem 140, p. 381 and Proof of Theorem XIII.98] that $\mu_{n}(k)$, the $n$th eigenvalue of $W_{0, Q}(k)$, is a continuous function of $k$, and we can choose measurable functions $\psi_{n}: \widetilde{Q} \rightarrow \mathcal{H}(k)$ such that $W_{0, Q}(k) \psi_{n}(k)=\mu_{n}(k) \psi_{n}(k)$ and $\left\{\psi_{n}(k)\right\}_{n=1,2, \ldots}$ is an orthonormal basis for $\mathcal{H}(k)$ for each $k$. We thus have

$$
W_{0, Q}(k)=\sum_{n=1}^{\infty} \mu_{n}(k) P_{n}(k),
$$

where $P_{n}(k)$ denotes the orthogonal projection on the vector $\psi_{n}(k)$ and the series converges strongly in the domain of $\widehat{W}_{0}$. Moreover, if $z \notin \sigma\left(W_{0}\right)$, say $\operatorname{dist}\left(z, \sigma\left(W_{0}\right)\right) \geq$ $\delta>0$, we have $\operatorname{dist}\left(z, \sigma\left(W_{0, Q}(k)\right)\right) \geq \delta$ for all $k$ and

$$
\widehat{\breve{R}_{0}(z)}=\int_{\widetilde{Q}}^{\oplus} \breve{R}_{0, Q}(k ; z) d k, \quad \text { with } \breve{R}_{0, Q}(k ; z)=\sum_{n=1}^{\infty} \frac{1}{\mu_{n}(k)-z} P_{n}(k) .
$$

Thus

$$
\left|\widehat{\breve{R}_{0}(z)}\right|=\int_{\widetilde{Q}}^{\oplus}\left|\breve{R}_{0, Q}(k ; z)\right| d k
$$

where $\left|\breve{R}_{0, Q}(k ; z)\right|$ is the operator on $L^{2}\left(Q ; \mathbb{C}^{\nu}\right)$ given by

$$
\left|\breve{R}_{0, Q}(k ; z)\right|=\sum_{n=1}^{\infty} \tau_{n}(k ; z) P_{n}(k) \quad \text { with } \tau_{n}(k ; z)=\frac{1}{\left|\mu_{n}(k)-z\right|} .
$$

We also introduce

$$
\left|\widehat{\breve{R}_{0, N}(z)}\right|=\int_{\widetilde{Q}}^{\oplus}\left|\breve{R}_{0, Q, N}(k ; z)\right| d k \quad \text { with } \quad\left|\breve{R}_{0, Q, N}(k ; z)\right|=\sum_{n=1}^{N} \tau_{n}(k ; z) P_{n}(k)
$$

where $N=1,2, \ldots$. Note that $\left|\breve{R}_{0, N}(z)\right| \rightarrow\left|\breve{R}_{0}(z)\right|$ strongly as $N \rightarrow \infty$, and for any $\Phi \in L^{2}\left(\mathbb{R}^{d} ; \mathbb{C}^{\nu}\right)$ we have

$$
\left\|\left|\breve{R}_{0, N}(z)\right| \Phi\right\|^{2}=\int_{\widetilde{Q}}\left\{\sum_{n=1}^{N} \tau_{n}(k ; z)^{2}\left|\left\langle\psi_{n}(k), \widehat{\Phi}(k)\right\rangle\right|^{2}\right\} d k \nearrow\left\|\left|\breve{R}_{0}(z)\right| \Phi\right\|^{2},
$$

as $N \rightarrow \infty$.

We recall that $\chi_{0}$ is the characteristic function of $Q$. As a multiplication operator it satisfies

$$
\begin{aligned}
{\left[\widehat{\chi_{0}} \widehat{\varphi}\right](k, x)=\widehat{\chi_{0} \varphi}(k, x) } & =\left(\frac{1}{2 \pi}\right)^{d / 2} e^{i k \cdot x} \varphi(x) \\
& =\left(\frac{1}{2 \pi}\right)^{d} \int_{\widetilde{Q}} e^{i\left(k-k^{\prime}\right) \cdot x} \widehat{\varphi}\left(k^{\prime}, x\right) d k^{\prime},
\end{aligned}
$$

for any $\varphi \in L^{2}\left(\mathbb{R}^{d} ; \mathbb{C}^{\nu}\right)$, with $x \in Q, k \in \tilde{Q}$. We set $K(z)=\chi_{0}\left|\breve{R}_{0}(z)\right| \chi_{0}, K_{N}(z)=$ $\chi_{0}\left|\breve{R}_{0, N}(z)\right| \chi_{0}$. Putting together (47) and (49), we can see that $K_{N}(z)$ is a bounded integral operator on $L^{2}\left(\tilde{Q} \times Q ; \mathbb{C}^{\nu}\right) \cong L^{2}\left(\tilde{Q} ; L^{2}\left(Q ; \mathbb{C}^{\nu}\right)\right)$ with kernel

$$
K_{N}(z)(k, x ; p, y)=\left(\frac{1}{2 \pi}\right)^{2 d} e^{i(k \cdot x-p \cdot y)} \int_{\widetilde{Q}}\left\{\sum_{n=1}^{N} \tau_{n}(q ; z) \psi_{n}(q, x) \overline{\psi_{n}(q, y)}\right\} d q
$$


The following lemma will help us separate the contributions of different bands. We use $\|T\|_{2}=\left(\operatorname{Tr} T^{*} T\right)^{1 / 2}$ to denote the Hilbert-Schmidt norm of an operator $T$.

LEMMA 3.1.

(i) For each $N=1,2, \ldots$ we have

$$
\left\|K_{N}(z)\right\|_{2}^{2}=\left(\frac{1}{2 \pi}\right)^{2 d} \sum_{n, m=1}^{N} \int_{\widetilde{Q}} \int_{\widetilde{Q}} \tau_{n}(k ; z) \tau_{m}(p ; z)\left|\left\langle\psi_{n}(k), \psi_{m}(p)\right\rangle\right|^{2} d k d p
$$

(ii) We have

$$
\|K(z)\|_{2} \leq \liminf _{N \rightarrow \infty}\left\|K_{N}(z)\right\|_{2} .
$$

(iii) If $d=3$ and $z=-1$, we have

$$
\|K(-1)\|_{2}^{2}=\left(\frac{1}{2 \pi}\right)^{6} \sum_{n, m=1}^{\infty} \int_{\widetilde{Q}} \int_{\widetilde{Q}} \tau_{n}(k ;-1) \tau_{m}(p ;-1)\left|\left\langle\psi_{n}(k), \psi_{m}(p)\right\rangle\right|^{2} d k d p
$$

Proof. It follows from (50) that

$$
\left\|K_{N}(z)\right\|_{2}^{2}=\int_{\widetilde{Q} \times Q} \int_{\widetilde{Q} \times Q}\left|K_{N}(z)(k, x ; p, y)\right|^{2} d p d y d k d x
$$

from which (51) follows by an explicit computation.

Since $K_{N}(z) \rightarrow K(z)$ strongly as $N \rightarrow \infty$, (52) follows from Fatou's lemma.

To prove (53), first note it follows from (48) that

$$
\left\|\chi_{0}\left|\breve{R}_{0, N}(z)\right| \chi_{0} \Phi\right\| \leq\left\|\left|\breve{R}_{0, N}(z)\right| \chi_{0} \Phi\right\| \leq\left\|\breve{R}_{0}(z) \chi_{0} \Phi\right\|
$$

for any $\Phi \in L^{2}\left(\mathbb{R}^{d} ; \mathbb{C}^{\nu}\right)$, so we can conclude that

$$
\sup _{N}\left\|K_{N}(z)\right\|_{2} \leq\left\|\left|\breve{R}_{0}(z)\right| \chi_{0}\right\|_{2} .
$$

We now restrict ourselves to $d=3$ and $z=-1$. In this case $\left|\breve{R}_{0}(-1)\right|=\breve{R}_{0}(-1)$ and the operator $\breve{R}_{0}(-1) \chi_{0}$ is Hilbert-Schmidt [FK3, Proposition 42], [FK5, Theorem 18]. We have $\tau_{n}(k ;-1) \geq 1>0$, so it follows from (56) that the series in the right-hand side of (53) is absolutely convergent. As $\left\|K_{N}(-1)-K_{M}(-1)\right\|_{2}^{2}$ is given by the obvious analogue of (51), we can conclude that the $K_{N}(-1)$ form a Cauchy sequence in the Hilbert-Schmidt norm; since $K_{N}(-1) \rightarrow K(-1)$ strongly, it follows that $\left\|K_{N}(-1)-K(-1)\right\|_{2} \rightarrow 0$, so (53) follows from (51).

We are now ready to prove Theorem 2.4; we will do so for the left edge $a$, the proof for the right edge $b$ being similar. So let $d=3$ and let $n_{1} \leq n_{0}$ be as in the discussion below Definition 2.2 and let $\eta \in(0, b-a)$. We have

$$
\begin{aligned}
& \quad K(a+\eta)= \\
& K_{n_{1}-1}(a+\eta)+\left\{K_{n_{0}}(a+\eta)-K_{n_{1}-1}(a+\eta)\right\}+\left\{K(a+\eta)-K_{n_{0}}(a+\eta)\right\} .
\end{aligned}
$$

Using (51) we get

$$
\left\|K_{n_{1}-1}(a+\eta)\right\|_{2} \leq\left(\frac{n_{1}-1}{c_{n_{1}-1}}\right)
$$


where

$$
c_{n_{1}-1}=\min _{n=1, \ldots, n_{1}-1}\left(a-\max _{k \in \widetilde{Q}} \mu_{n}(k)\right)>0
$$

by the definition of $n_{1}$. If $n>n_{0}$ we have

$$
\begin{aligned}
\tau_{n}(k ; a+\eta)=\frac{\mu_{n}(k)+1}{\mu_{n}(k)-(a+\eta)} \tau_{n}(k ;-1) & \leq\left(1+\frac{1+a}{\mu_{n}(k)-(a+\eta)}\right) \tau_{n}(k ;-1) \\
& \leq\left(1+\frac{1+a}{b-(a+\eta)}\right) \tau_{n}(k ;-1),
\end{aligned}
$$

so it follows from (52), (51), (53), and (56) that

$$
\limsup _{\eta \rightarrow 0} \| K(a+\eta)-K_{n_{0}}\left(a+\eta\left\|_{2} \leq\left(1+\frac{1+a}{b-a}\right)\right\| \breve{R}_{0}(-1) \chi_{0} \|_{2}<\infty .\right.
$$

Finally, it follows from (51) and Assumption (25) that

$$
\limsup _{\eta \rightarrow 0}\left\|K_{n_{0}}(a+\eta)-K_{n_{1}-1}(a+\eta)\right\|_{2} \leq\left(\frac{1}{2 \pi}\right)^{3} \sum_{n=n_{1}-1}^{n_{0}} \int_{\widetilde{Q}} \frac{1}{a-\mu_{n}(k)} d k<\infty .
$$

Combining (57), (58), (59), (61), and (62) we get lim $\sup _{\eta \rightarrow 0}\|K(a+\eta)\|_{2}<\infty$, which is the same as (26), so Theorem 2.4 is proven.

4. Bounded, relatively compact perturbations. Let $H_{g}=H_{0}+g V$, where $H_{0}$ and $V$ are self-adjoint operators, $H_{0}$ has a gap $(a, b)$ in its spectrum, $V \geq 0$ is bounded and relatively compact with respect to $H_{0}$, and $\mathrm{g}$ is a real number. By [RS4, Section XIII.4, Corollary 2], we have $\sigma_{\text {ess }}\left(H_{g}\right)=\sigma_{\text {ess }}\left(H_{0}\right)$, so the spectrum of the operator $H_{g}$ in the gap $(a, b)$ consists of isolated eigenvalues of finite multiplicity, which can only accumulate at the edges $a$ and $b$.

We now apply the Birman-Schwinger method. The eigenvalue problem

$$
H_{0} \psi+g V \psi=\lambda \psi, \quad \lambda \in(a, b)
$$

can be rewritten as

$$
\psi=-g R_{0}(\lambda) V \psi, \quad \text { where } R_{0}(\lambda)=\left(H_{0}-\lambda I\right)^{-1} .
$$

If we set

$$
\mathcal{R}(\lambda)=\mathcal{R}_{H_{0}, V}(\lambda)=-\sqrt{V} R_{0}(\lambda) \sqrt{V}
$$

we obtain

$$
\mathcal{R}(\lambda) \varphi=\frac{1}{g} \varphi \text { for } \varphi=\sqrt{V} \psi
$$

The Birman-Schwinger operator $\mathcal{R}(\lambda)$ is a compact self-adjoint operator. We have the following lemma $[\mathrm{Kl}]$.

LEMma 4.1. Let $g \models 0$. Then $\lambda \in(a, b)$ is an eigenvalue of $H_{g}$ with multiplicity $m$ if and only if $1 / g$ is an eigenvalue of $\mathcal{R}(\lambda)$ with multiplicity $m$. 
Proof. We give the proof for completeness. Let $\lambda \in(a, b)$ be an eigenvalue for $H_{g}$ with eigenfunction $\psi$, i.e., (63) holds with $\Psi \nRightarrow 0$. It follows from (64)-(66) that $1 / g$ is an eigenvalue of $\mathcal{R}(\lambda)$ with eigenfunction $\varphi=\sqrt{V} \psi$. Since $\psi=-g R_{0}(\lambda) \sqrt{V} \varphi$, we clearly have $m_{\lambda} \leq m_{1 / g}$, where $m_{\lambda}$ and $m_{1 / g}$ denote the multiplicity of $\lambda$ and $1 / g$ as eigenvalues of $H_{g}$ and $\mathcal{R}(\lambda)$, respectively.

Conversely, if $1 / g$ is an eigenvalue of $\mathcal{R}(\lambda)$ with eigenfunction $\varphi$, then if we set $\psi=-g R_{0}(\lambda) \sqrt{V} \varphi$, we have $\sqrt{V} \psi=g \mathcal{R}(\lambda) \varphi=\varphi$, so we get $\left(H_{0}-\lambda I\right) \psi=$ $-g \sqrt{V} \varphi=g V \psi$, which is the same as (63). It also follows that $m_{1 / g} \leq m_{\lambda}$.

Given $\lambda$ in the gap $(a, b)$, let $r_{1}^{+}(\lambda) \geq r_{2}^{+}(\lambda) \geq \cdots \geq 0$ and $r_{1}^{-}(\lambda) \leq r_{2}^{-}(\lambda) \leq$ $\cdots \leq 0$ be infinite sequences of, respectively, the positive and negative eigenvalues of the operator $\mathcal{R}(\lambda)$. If we have a finite number of either positive or negative eigenvalues, we complete the sequence by assigning the value 0 . The sequence $r_{n}^{-}(\lambda)$, $n=1,2, \ldots$, can be obtained by applying the min-max principle to the operator $\chi_{(-\infty, 0]}(\mathcal{R}(\lambda)) \mathcal{R}(\lambda)$; similarly, we obtain $-r_{n}^{+}(\lambda), n=1,2, \ldots$, by applying the min-max principle to the operator $-\chi_{[0, \infty)}(\mathcal{R}(\lambda)) \mathcal{R}(\lambda)$.

It turns out that it is more convenient to first study the dependence of the eigenvalues $r_{n}^{ \pm}(\lambda)$ of the operator $\mathcal{R}(\lambda)$ on the parameter $\lambda$, and then use Lemma 4.1 to obtain information about the eigenvalues of $H_{g}$ in the gap $(a, b)$ (see [Kl, ADH]).

LEMma 4.2. The functions $r_{n}^{ \pm}(\lambda)$ are monotonically decreasing, continuous functions of $\lambda \in(a, b)$ for each $n=1,2, \ldots$.

Proof. The lemma follows from the fact that the the map $\lambda \in(a, b) \rightarrow \mathcal{R}(\lambda)$ is norm continuous and operator monotone decreasing.

The following proposition is an immediate consequence of Lemmas 4.1 and 4.2; it gives a criterion for the absence of eigenvalues of $H_{g}$ in the gap $(a, b)$ for small $g$.

Proposition 4.3. The following statements are equivalent:

(i) The operator $H_{g}$ has no eigenvalues in the gap $(a, b)$ for small $g$.

(ii) We have

$$
\max _{\lambda \in(a, b)}\|\mathcal{R}(\lambda)\|=\max \left\{r_{1}^{+}(a+0),-r_{1}^{-}(b-0)\right\}<\infty .
$$

We can summarize some important conclusions of the Birman-Schwinger method in the following theorem, which is an immediate consequence of Lemma 4.1 (including the considerations right before it) and Lemma 4.2.

THEOREM 4.4. Let $H_{0}$ and $V$ be self-adjoint operators. Suppose that $H_{0}$ has a gap $(a, b)$ in its spectrum and $V$ is bounded, relatively compact with respect to $H_{0}$; set $H=H_{0}+V$. Then:

(i) If $V \geq 0$, the only possible point of accumulation of $\sigma(H) \cap(a, b)$ is a. In this case the eigenvalues $\lambda_{1} \geq \lambda_{2} \geq \cdots$ of the operator $H$ in the gap $(a, b)$ coincide with the set of the solutions of the equations

$$
r_{n}^{+}(\lambda)=1, \quad n=1,2, \ldots,
$$

where $r_{n}^{+}(\lambda)$ are the positive eigenvalues of the operator $\mathcal{R}(\lambda)$ defined by (65). Moreover, if $\varphi(\lambda)$ is an eigenmode of the operator $\mathcal{R}(\lambda)$ with eigenvalue $r_{n}^{+}(\lambda)=1$, then

$$
\psi=\left(H_{0}-\lambda I\right)^{-1} \sqrt{V} \varphi(\lambda)
$$

is an exponentially localized eigenmode of the operator $H$ with eigenvalue $\lambda$.

(ii) If $V \leq 0$, the only possible point of accumulation of $\sigma(H) \cap(a, b)$ is $b$. In this case the eigenvalues $\lambda_{1} \leq \lambda_{2} \leq \cdots$ of the operator $H$ in the gap $(a, b)$ coincide 
with the set of the solutions of the equations

$$
r_{n}^{-}(\lambda)=-1, \quad n=1,2, \ldots,
$$

where $r_{n}^{-}(\lambda)$ are the negative eigenvalues of the operator $\mathcal{R}(\lambda)$ defined by $(65)$. Moreover, if $\varphi(\lambda)$ is an eigenmode of the operator $\mathcal{R}(\lambda)$ with eigenvalue $r_{n}^{-}(\lambda)=-1$, then

$$
\psi=-\left(H_{0}-\lambda I\right)^{-1} \sqrt{-V} \varphi(\lambda)
$$

is an exponentially localized eigenmode of the operator $H$ with eigenvalue $\lambda$.

In order to estimate the number of eigenvalues of the operator $H=H_{0}+V$ in the gap $(a, b)$, we introduce a counting function $\mathcal{N}_{H_{0}, V}^{+}$, which will count the eigenvalues generated in the gap. We start with a descriptive definition which will be followed by a rigorous one. We pick a number $0<\eta<(b-a)$ and put an observer at the point $a+\eta$. As $g$ changes gradually from 0 to 1 , the operator $H_{g}$ develops eigenvalues in the gap which split off the endpoint $a$ and move toward the point $b$. The observer counts how many times an eigenvalue of $H_{g}$ passes through the point $a+\eta$ as $g$ changes from 0 to 1 ; the number of such events as a function of $a+\eta$ will be called the (left-edge) counting function and denoted by $\mathcal{N}_{H_{0}, V}^{+}(a+\eta)$.

Definition 4.5. For $0<\eta<(b-a)$ we define the (left-edge) counting function $\mathcal{N}_{H_{0}, V}^{+}(a+\eta)$ as the number of $g^{\prime} \in(0,1]$, such that $a+\eta$ is an eigenvalue of $H_{g^{\prime}}$. (If $a+\eta$ is an eigenvalue of $H_{g^{\prime}}$ of multiplicity $m, g^{\prime}$ must be counted $m$ times.)

Similarly, we define the (right-edge) counting function $\mathcal{N}_{H_{0}, V}(b-\eta)$ as the number of $g^{\prime} \in[-1,0)$, such that $b-\eta$ is an eigenvalue of $H_{g^{\prime}}$.

The counting function can be estimated as follows.

LEMMA 4.6. Letting $0<\eta<(b-a)$, we have

$$
\mathcal{N}_{H_{0}, V}^{+}(a+\eta) \leq \operatorname{Tr}\left(\left\{\mathcal{R}_{H_{0}, V}(a+\eta)\right\}^{2}\right)
$$

and

$$
\mathcal{N}_{H_{0}, V}^{-}(b-\eta) \leq \operatorname{Tr}\left(\left\{\mathcal{R}_{H_{0}, V}(b-\eta)\right\}^{2}\right)
$$

Proof. It follows from Lemma 4.1 that

$$
\begin{aligned}
\mathcal{N}_{H_{0}, V}^{+}(a+\eta) & =\#\left\{\mu \geq 1 ; \mu \text { eigenvalue of } \mathcal{R}_{H_{0}, V}(a+\eta)\right\} \\
& \leq \operatorname{Tr}\left(\left\{\mathcal{R}_{H_{0}, V}(a+\eta)\right\}^{2}\right) .
\end{aligned}
$$

The counting function can be used to estimate the number of eigenvalues of $H$ in the gap.

LEMMA 4.7. Letting $0<\eta<(b-a)$, we have

$$
N_{H_{0}+V}(a+\eta, b) \leq \mathcal{N}_{H_{0}, V}^{+}(a+\eta)
$$

and

$$
N_{H_{0}-V}(a, b-\eta) \leq \mathcal{N}_{H_{0}, V}^{-}(b-\eta)
$$


Proof. We have

$$
\begin{aligned}
N_{H_{0}+V}(a+\eta, b) & =\#\left\{\lambda \in(a+\eta, b) ; \lambda \text { eigenvalue of } H_{0}+V\right\} \\
& =\#\left\{\lambda \in(a+\eta, b) ; 1 \text { eigenvalue of } \mathcal{R}_{H_{0}, V}(\lambda)\right\} \\
& \leq \#\left\{\mu \geq 1 ; \mu \text { eigenvalue of } \mathcal{R}_{H_{0}, V}(a+\eta)\right\}=\mathcal{N}_{H_{0}, V}^{+}(a+\eta),
\end{aligned}
$$

where (78) follows from Lemma 4.1 and the inequality in (79) is a consequence of Lemma 4.2 .

The counting functions have the important property of being monotone in the operator $V$ [ADH, Corollary 1.7].

LEMmA 4.8. Let $V_{2} \geq V_{1} \geq 0$ be bounded and relatively compact with respect to $H_{0}$. Then

$$
\mathcal{N}_{H_{0}, V_{1}}^{+}(a+\eta) \leq \mathcal{N}_{H_{0}, V_{2}}^{+}(a+\eta)
$$

and

$$
\mathcal{N}_{H_{0}, V_{1}}^{-}(b-\eta) \leq \mathcal{N}_{H_{0}, V_{2}}^{-}(b-\eta) .
$$

Proof. The statement follows from (74) and Theorem A.2.

5. Reduction to compact perturbations. If the medium described by $\varepsilon(x)$ (as in (4)) was obtained from the background medium, described by a function $\varepsilon_{0}(x)$ as in (4) - not necessarily periodic - by the insertion of a defect, we can write $W=$ $W_{0}+Q$, but the perturbation $Q$ is unbounded. This can be remedied by working with the resolvents.

Proposition 5.1. Suppose $d \leq 3$. Let $\varepsilon(x)$ and $\varepsilon_{0}(x)$, as in (4), differ by a defect. If we set $H=(W+I)^{-1}$ and $H_{0}=\left(W_{0}+I\right)^{-1}$, then $V=H-H_{0}$ is a Hilbert-Schmidt operator.

Proof. Let $\Theta(x)=1 / \varepsilon(x)-1 / \varepsilon_{0}(x)$; by our hypotheses it is a bounded measurable function with compact support. We clearly have $Y \equiv W-W_{0}=\left(\nabla^{\sharp}\right)^{*} \Theta \nabla^{\sharp}$ as quadratic forms on the common quadratic form domain of $W$ and $W_{0}$, where $\nabla^{\sharp}=\nabla$ if $W=A$ and $\nabla^{\sharp}=\nabla^{\times}$if $W=M$. Thus, $V=-H V H_{0}=-T^{*} \Theta T_{0}$, with $T=\chi \nabla^{\sharp} H$ and $T_{0}=\chi \nabla^{\sharp} H_{0}$, where $\chi$ is the characteristic function of an open, bounded set containing the support of $\Theta$.

Since $T$ and $T_{0}$ are Hilbert-Schmidt operators by [FK4, Lemma 6], we can conclude that $V$ is Hilbert-Schmidt.

Proposition 5.1 allows us to define counting functions for the operator $W$. Note that $\left((b+1)^{-1},(a+1)^{-1}\right)$ is a gap in the spectrum of $H_{0}$ if $(a, b)$ is a gap in the spectrum of $W_{0}$.

Definition 5.2. Let $W, W_{0}, H, H_{0}, V$ be as in Proposition 5.1. Suppose $(a, b)$ is a gap in the spectrum of $W_{0}$, and let $0<\eta \leq b-a$. If the defect is positive, we define the left-edge counting function for $W$ by

$$
\mathcal{N}_{W}^{+}(a+\eta)=\mathcal{N}_{H_{0}, V}^{-}\left((a+1+\eta)^{-1}\right) .
$$

Similarly, if the defect is negative, we define the right-edge counting function for $W$ by

$$
\mathcal{N}_{W}^{-}(b-\eta)=\mathcal{N}_{H_{0}, V}^{+}\left((b+1-\eta)^{-1}\right)
$$


Lemmas 4.7 and 4.8 have the following immediate counterparts for acoustic and Maxwell operators.

Lemma 5.3. Suppose $d \leq 3$. Let $\varepsilon(x)$ and $\varepsilon_{0}(x)$, as in (4) and (30), differ by a defect. Assume $(a, b)$ is a gap in the spectrum of $W_{0}$, and let $0<\eta \leq b-a$. If the defect is positive we have

$$
N_{W}(a+\eta, b) \leq \mathcal{N}_{W}^{+}(a+\eta) .
$$

Similarly, if the defect is negative we have

$$
N_{W}(a, b-\eta) \leq \mathcal{N}_{W}^{-}(b-\eta) .
$$

Lemma 5.4. Suppose $d \leq 3$. Let $\varepsilon_{i}(x), i=0,1,2$, be as in (4), differing from each other by a defect, and let $W_{i}, i=0,1,2$, be the corresponding operators. Assume $(a, b)$ is a gap in the spectrum of $W_{0}$, and let $0<\eta \leq b-a$. Then, if $\varepsilon_{2}(x) \leq \varepsilon_{1}(x) \leq \varepsilon_{0}(x)$ we have

$$
\mathcal{N}_{W_{1}}^{+}(a+\eta) \leq \mathcal{N}_{W_{2}}^{+}(a+\eta) .
$$

Similarly, if $\varepsilon_{2}(x) \geq \varepsilon_{1}(x) \geq \varepsilon_{0}(x)$ we have

$$
\mathcal{N}_{W_{1}}^{-}(b-\eta) \leq \mathcal{N}_{W_{2}}^{-}(b-\eta) .
$$

6. Smooth defects. We call a defect smooth if the function $\theta(x)$ in $(30)$ is continuously differentiable with compact support. In this section we will prove some lemmas concerning smooth defects. Theorem 2.5 is proved in the next section by a reduction to the case of smooth defects.

Lemma 6.1. Suppose $d \leq 3$. Let $\varepsilon(x)$ and $\varepsilon_{0}(x)$, as in (4) and (30), differ by either a positive or negative smooth defect, and set $\chi$ to be the characteristic function of an open, bounded set containing the support of $\theta$. Suppose $(a, b)$ is a gap in the spectrum of $W_{0}$, and let $0<\eta \leq b-a$. Then:

(i) For a negative defect we have

$$
\mathcal{N}_{W}^{-}(b-\eta) \leq(b+1-\eta)^{2} \operatorname{Tr}\left(\chi \breve{R}_{0}(b-\eta) \breve{R}(-1) \widetilde{Y}^{2} \breve{R}(-1) \breve{R}_{0}(b-\eta) \chi\right) .
$$

(ii) For a positive defect we have

$$
\mathcal{N}_{W}^{+}(a+\eta) \leq(a+1+\eta)^{2} \operatorname{Tr}\left(\chi \breve{R}_{0}(a+\eta) \breve{R}(-1) \widetilde{Y}^{2} \breve{R}(-1) \breve{R}_{0}(a+\eta) \chi\right) .
$$

Proof. Let us suppose the smooth defect is negative, the other case being similar. We take $H, H_{0}, V$ as in Proposition 5.1, so $V \geq 0$ is a Hilbert-Schmidt operator. Thus, given $0<\eta \leq b-a$, we can apply Lemma 4.6 and (83) to conclude

$$
\begin{aligned}
\mathcal{N}_{W}^{-}(b-\eta) & \leq \operatorname{Tr}\left(\left\{\sqrt{V}\left(H_{0}-(b+1-\eta)^{-1} I\right)^{-1} \sqrt{V}\right\}^{2}\right) \\
& =\operatorname{Tr}\left(\left\{V\left(H_{0}-(b+1-\eta)^{-1} I\right)^{-1}\right\}^{2}\right) .
\end{aligned}
$$


But

$$
\begin{aligned}
\left(H_{0}-(b+1-\eta)^{-1} I\right)^{-1} & =-(b+1-\eta)\left(W_{0}+I\right)\left(W_{0}-(b-\eta) I\right)^{-1} \\
& =-(b+1-\eta)\left(W_{0}+I\right) R_{0}(b-\eta)
\end{aligned}
$$

so

$$
\begin{aligned}
V\left(H_{0}-(b+1-\eta)^{-1} I\right)^{-1} & =-(b+1-\eta)(W+I)^{-1} Y R_{0}(b-\eta) \\
& =-(b+1-\eta) R(-1) Y R_{0}(b-\eta) .
\end{aligned}
$$

Note that $\chi \breve{R}_{0}(b-\eta)$ is Hilbert-Schmidt (see [FK3, Proposition 42] and [FK5, Theorem 18]), and since the defect is smooth, $R(-1) Y$ is a bounded operator (see [FK4, Proof of Lemma 6]). Thus

$$
\begin{aligned}
\mathcal{N}_{W}^{-}(b-\eta) & \leq(b+1-\eta)^{2} \operatorname{Tr}\left(\left\{R(-1) Y \chi R_{0}(b-\eta)\right\}^{2}\right) \\
& =(b+1-\eta)^{2} \operatorname{Tr}\left(\left\{\breve{R}(-1) \widetilde{Y} \chi \breve{R}_{0}(b-\eta)\right\}^{2}\right) \\
& =(b+1-\eta)^{2} \operatorname{Tr}\left(\left\{\chi \breve{R}_{0}(b-\eta) \breve{R}(-1) \widetilde{Y}\right\}^{2}\right) \\
& \leq(b+1-\eta)^{2} \operatorname{Tr}\left(\chi \breve{R}_{0}(b-\eta) \breve{R}(-1) \widetilde{Y}^{2} \breve{R}(-1) \breve{R}_{0}(b-\eta) \chi\right)
\end{aligned}
$$

where we used the inequality $\left|\operatorname{Tr} B^{2}\right| \leq \operatorname{Tr} B^{*} B$ to get (98).

The lemma is proven.

We now need to estimate from above the positive operator $\breve{R}(-1) \widetilde{Y}^{2} \breve{R}(-1)$. To do so we pick $\delta>0$ and introduce the positive self-adjoint operator $\Gamma=\Gamma_{\delta}$ given by multiplication by the function

$$
\gamma(x)=\gamma_{\delta}(x)=\sum_{m \in \mathbb{Z}^{d}} \gamma_{m} \chi_{m}(x), \text { with } \gamma_{m}=(1+|m|)^{d+\delta / 2} .
$$

Note that

$$
\gamma_{m+n} \leq \gamma_{m} \gamma_{n} \text { for all } m, n \in \mathbb{Z}^{d} .
$$

Lemma 6.2. Let $\varepsilon(x)$ and $\varepsilon_{0}(x)$, as in (4), (30), and (32), differ by a smooth defect, with $\theta(x)$ vanishing outside a cube $\Lambda_{\ell}$ of side $\ell>0$ centered at 0 . Suppose $d \leq 3$ and let $0<\delta \leq 1$. Then there exists a finite constant $K$, such that

$$
\breve{R}(-1) \widetilde{Y}^{2} \breve{R}(-1) \leq C\|\theta\|_{\infty}^{2}(\ell+2)^{2 d+\delta} \Gamma_{\delta}^{-2},
$$

with

$$
C=\left\{1+K\left[1+\varepsilon_{-}^{-(2 d+\delta)}\right]^{1 / 2}\left[1+\frac{\sqrt{\varepsilon_{+}}}{\varepsilon_{0,-}}\left(\sqrt{\varepsilon_{-}}+\frac{1}{\sqrt{\varepsilon_{-}}}\right) \frac{\|\nabla \theta\|_{\infty}}{\|\theta\|_{\infty}}\right]\right\}^{2}
$$

Proof. In view of Corollary C.2 it suffices to estimate $\|\tilde{Y} \breve{R}(-1) \Gamma\|_{\mathcal{F}}$. (See C for the notation.) Let us consider the case $W=A$, the case $W=\mathbf{M}$ being similar. In this case

$$
Y=-\nabla \cdot \frac{\theta(x)}{\varepsilon_{0}(x)} \nabla=\theta W-\frac{1}{\varepsilon_{0}}(\nabla \theta) \cdot \nabla
$$


hence,

$$
\widetilde{Y} \breve{R}(-1)=\theta-\theta \breve{R}(-1)-\frac{1}{\varepsilon_{0}}(\nabla \theta) \cdot \nabla \breve{R}(-1) .
$$

Clearly,

$$
\|\theta \Gamma\|_{\mathcal{F}} \leq\|\theta\|_{\infty}(\ell+2)^{d / 2}\left(1+\frac{\sqrt{d}}{2}(\ell+1)\right)^{d+\delta / 2} \leq\|\theta\|_{\infty}(\ell+2)^{d+\delta / 2},
$$

where we used $d \leq 3$. A straightforward calculation using Proposition B.1 and (100) gives

$$
\begin{aligned}
\|\theta \breve{R}(-1) \Gamma\|_{\mathcal{F}} & \leq\|\theta\|_{\infty}\left[\sum_{\substack{n \in \mathbb{Z}^{d} \cap \wedge_{\ell+1} \\
m \in \mathbb{Z}^{d}}}\left\|\chi_{n} \breve{R}(-1) \chi_{m}\right\|^{2} \gamma_{m}^{2}\right]^{1 / 2} \\
& \leq \alpha_{d}\|\theta\|_{\infty}\left[\sum_{\substack{n \in \mathbb{Z}^{d} \cap \Lambda_{\ell+1} \\
m \in \mathbb{Z}^{d}}} \mathrm{e}^{-2 \beta|m-n|}(1+|m|)^{d+\delta}\right]^{1 / 2} \\
& \leq \alpha_{d}\|\theta\|_{\infty}(\ell+2)^{d+\delta / 2}\left[\sum_{m \in \mathbb{Z}^{d}} \mathrm{e}^{-2 \beta|m|}(1+|m|)^{d+\delta}\right]^{1 / 2} \\
& \leq K_{1}\|\theta\|_{\infty}(\ell+2)^{d+\delta / 2}\left[1+\beta^{-(2 d+\delta)}\right]^{1 / 2} \\
& \leq K\|\theta\|_{\infty}(\ell+2)^{d+\delta / 2}\left[1+\varepsilon_{-}^{-(2 d+\delta)}\right]^{1 / 2}
\end{aligned}
$$

where $\alpha_{d}$ and $\beta$ are as in Proposition B.1, and $K_{1}, K$ are finite constants, independent of all parameters for $d \leq 3$ and $0<\delta \leq 1$. Similarly,

$$
\begin{aligned}
& \left\|\frac{1}{\varepsilon_{0}}(\nabla \theta) \cdot \nabla \breve{R}(-1) \Gamma\right\|_{\mathcal{F}} \\
& \leq K\left[1+\varepsilon_{-}^{-(2 d+\delta)}\right]^{1 / 2} \frac{\sqrt{\varepsilon_{+}}}{\varepsilon_{0,-}}\left(\sqrt{\varepsilon_{-}}+\frac{1}{\sqrt{\varepsilon_{-}}}\right)\|\nabla \theta\|_{\infty}(\ell+2)^{d+\delta / 2} .
\end{aligned}
$$

The estimate (101) follows from (105), (109), (110), and Corollary C.2.

7. Regular edges. We are now ready to prove Theorem 2.5. We will consider the case when the right edge $b$ is regular and the defect is negative, the other case being analogous. Thus $\theta(x) \leq 0$, vanishing outside a cube $\Lambda_{\ell}$ of side $\ell>0$, which we take to be centered at 0 without loss of generality. Note $\theta_{+}=0$ and $\varepsilon_{-}=\varepsilon_{0,-}$.

We start by reducing to the case of a smooth defect. We pick a continuously differentiable function $f(t)$ of one real variable, with $0 \leq f(t) \leq 1$ for all $t, f(t)=1$ for $|t| \leq \frac{\ell}{2}, f(t)=0$ for $|t| \geq \frac{\ell+1}{2}$, and $\left|f^{\prime}(t)\right| \leq 3$ for all $t$. (Such a function always exists.) We now set $\theta_{s}(x)=\theta_{-} \prod_{i=1}^{d} f\left(x_{i}\right)$; it is a continuously differentiable function vanishing outside the cube $\Lambda_{\ell+1}$ centered at 0 , with $\left\|\theta_{s}\right\|_{\infty}=\left|\theta_{-}\right|$and 
$\left\|\nabla \theta_{s}\right\|_{\infty} \leq 3 \sqrt{d}\left|\theta_{-}\right|$. Moreover, $\theta(x) \geq \theta_{s}(x)$ for all $x$. We set $\varepsilon_{s}(x)=\frac{\varepsilon_{0}(x)}{1+\theta_{s}(x)}$ and let $W_{s}$ denote the corresponding acoustic or Maxwell operator. Note $\varepsilon_{s, \pm}=\varepsilon_{ \pm}$ by (32). If $d \leq 3$ and $0<\delta \leq 1$ for any given $0<\eta \leq b-a$ and we get from Lemmas $5.3,5.4,6.1$, and 6.2 that

$$
\begin{aligned}
& N_{W}(a, b-\eta) \leq \mathcal{N}_{W_{s}}^{-}(b-\eta) \\
\leq & (b+1-\eta)^{2} C_{d, \varepsilon_{0, \pm}, \theta_{-}, \delta} \theta_{-}^{2}(\ell+3)^{2 d+\delta} \operatorname{Tr}\left(\chi \breve{R}_{0}(b-\eta) \Gamma_{\delta}^{-2} \breve{R}_{0}(b-\eta) \chi\right),
\end{aligned}
$$

where

$$
\begin{aligned}
& C_{d, \varepsilon_{0, \pm}, \theta_{-}, \delta} \\
& =\left\{1+K\left[1+\varepsilon_{0,-}^{-(2 d+\delta)}\right]^{1 / 2}\left[1+3 \sqrt{d} \frac{\sqrt{\varepsilon_{+}}}{\varepsilon_{0,-}}\left(\sqrt{\varepsilon_{0,-}}+\frac{1}{\sqrt{\varepsilon_{0,-}}}\right)\right]\right\}^{2},
\end{aligned}
$$

and $\chi$ is the characteristic function of the cube $\Lambda_{\ell+1}$.

Recalling (99), we see that

$$
\begin{aligned}
& \operatorname{Tr}\left(\chi \breve{\mathrm{R}}_{0}(\mathrm{~b}-\eta) \Gamma_{\delta}^{-2} \breve{\mathrm{R}}_{0}(\mathrm{~b}-\eta) \chi\right) \\
& =\sum_{m \in \mathbb{Z}^{d}}(1+|m|)^{-(d+\delta)} \operatorname{Tr}\left(\chi \breve{R}_{0}(b-\eta) \chi_{m} \breve{R}_{0}(b-\eta) \chi\right) .
\end{aligned}
$$

We have

$$
\operatorname{Tr}\left(\chi \breve{\mathrm{R}}_{0}(\mathrm{~b}-\eta) \chi_{\mathrm{m}} \breve{\mathrm{R}}_{0}(\mathrm{~b}-\eta) \chi\right)=\operatorname{Tr}\left(\chi_{\mathrm{m}} \breve{\mathrm{R}}_{0}(\mathrm{~b}-\eta) \chi \breve{\mathrm{R}}_{0}(\mathrm{~b}-\eta) \chi_{\mathrm{m}}\right)
$$

$$
\leq \sum_{n \in \mathbb{Z}^{d} \cap \Lambda_{\ell+2}} \operatorname{Tr}\left(\chi_{m} \breve{R}_{0}(b-\eta) \chi_{n} \breve{R}_{0}(b-\eta) \chi_{m}\right)
$$

$$
\leq 4 \sum_{n \in \mathbb{Z}^{d} \cap \Lambda_{\ell+2}}\left\{\operatorname{Tr}\left[\left(\chi_{m}\left|\breve{R}_{0}(b-\eta)\right| \chi_{m}\right)^{2}\right]\right\}^{1 / 2}\left\{\operatorname{Tr}\left[\left(\chi_{n}\left|\breve{R}_{0}(b-\eta)\right| \chi_{n}\right)^{2}\right]\right\}^{1 / 2}
$$

$$
\leq 4(\ell+3)^{d} \operatorname{Tr}\left[\left(\chi_{0}\left|\breve{R}_{0}(b-\eta)\right| \chi_{0}\right)^{2}\right]
$$

where (117) follows from Lemma D.1, and (118) is a consequence of the periodicity of the operator $\breve{R}_{0}(b-\eta)$.

Combining (111), (113), and (118) and using

$$
\sum_{m \in \mathbb{Z}^{d}}(1+|m|)^{-(d+\delta)} \leq \frac{K_{2}}{\delta}
$$

with a fixed constant $K_{2}$ for $d \leq 3$ and $0<\delta \leq 1$, we get

$$
\begin{aligned}
& N_{W}(a, b-\eta) \\
& \leq \frac{4 K_{2}}{\delta}(b+1-\eta)^{2} C_{d, \varepsilon_{0, \pm}, \theta_{-}, \delta} \theta_{-}^{2}(\ell+3)^{3 d+\delta} \operatorname{Tr}\left[\left(\chi_{0}\left|\breve{R}_{0}(b-\eta)\right| \chi_{0}\right)^{2}\right]
\end{aligned}
$$


so (35) follows.

Theorem 2.5 is proven.

Appendix A. Minimax principle in a spectral gap. We use the following version of the minimax principle.

THEOREM A.1. Let $A$ be a bounded from below self-adjoint operator, and let $\lambda_{1} \leq \lambda_{2} \leq \cdots$ be the set of the eigenvalues of $A$ (taking into account their multiplicity) in the half-interval $\left(-\infty, \inf \left\{\sigma_{\text {ess }}(A)\right\}\right)$. If the set of those eigenvalues is finite, i.e., for some finite positive integer $n$ the numbers $\lambda_{1} \leq \lambda_{2} \leq \cdots \leq \lambda_{n}$ are the only eigenvalues (taking in account the multiplicity) of the operator $A$ below $\inf \left\{\sigma_{\mathrm{ess}}(A)\right\}$, we set $\lambda_{n+1}=\lambda_{n+2}=\cdots=\inf \left\{\sigma_{\text {ess }}(A)\right\}$. For each $n=1,2, \ldots$, we have

$$
\lambda_{n}=\inf _{L \subset \mathcal{D}(A): \operatorname{dim} L=n}\left[\sup _{\psi \in L:\|\psi\|=1}\langle\psi, A \psi\rangle\right] .
$$

Proof. Let us denote by $\mu_{n}$ the right-hand side of (A.120) we must show $\mu_{n}=\lambda_{n}$ for all $n=1,2, \ldots$ It follows immediately from [RS4, Theorem XIII.3] that for $L \subset \mathcal{D}(A)$ with $\operatorname{dim} L=n$, we have

$$
\lambda_{n} \leq \sup _{\psi \in L:\|\psi\|=1}\langle\psi, A \psi\rangle, \text { so } \lambda_{n} \leq \mu_{n} .
$$

To prove the opposite inequality we must consider two separate cases. Let $\alpha_{0}=\inf \left\{\sigma_{\text {ess }}(A)\right\}$. If $\lambda_{n}<\alpha_{0}$, we introduce the space $L_{n}$ which is the span of the eigenvectors corresponding to the eigenvalues $\lambda_{1} \leq \cdots \leq \lambda_{n}$, note $\operatorname{dim} L=n$. It then follows from the definition of $\mu_{n}$ that

$$
\mu_{n} \leq \sup _{\psi \in L_{n}:\|\psi\|=1}\langle\psi, A \psi\rangle=\lambda_{n} .
$$

If $\lambda_{n}=\alpha_{0}$, we have $\operatorname{Tr} \chi_{\left[\alpha_{0}, \alpha_{0}+\delta\right)}(A)=\infty$ for any $\delta>0$. This means that given any $\delta>0$, we can pick $L \subset \mathcal{D}(A)$ with $\operatorname{dim} L=n$, such that $\sup _{\psi \in L:\|\psi\|=1}\langle\psi, A \psi\rangle \leq$ $\alpha_{0}+\delta$, so $\mu_{n} \leq \alpha_{0}+\delta$. Thus $\mu_{n} \leq \alpha_{0}=\lambda_{n}$.

Now for a compact self-adjoint operator $A$ let $\lambda_{1}^{+}(A) \geq \lambda_{2}^{+}(A) \geq \cdots \geq 0$ and $\lambda_{1}^{-}(A) \leq \lambda_{2}^{-}(A) \leq \cdots \leq 0$ be infinite sequences of, respectively, the positive and negative eigenvalues of the operator $A$. If we have a finite number of either positive or negative eigenvalues, we complete the sequence by assigning the value 0 . The sequence $\lambda_{n}^{-}(A), n=1,2, \ldots$, can be obtained by applying the min-max principle to the operator $\chi_{(-\infty, 0]}(A) A$; similarly, we obtain $-\lambda_{n}^{+}(A), n=1,2, \ldots$, by applying the min-max principle to the operator $-\chi_{[0, \infty)}(A) A$. We have the following application of the minimax principle. (See $[\mathrm{ADH}$, Proposition 1.6]; we give a proof based on Theorem A.1.)

TheOREM A.2. Let $A$ be a bounded self-adjoint operator, and let $V_{2} \geq V_{1} \geq 0$ be bounded and relatively compact with respect to $A$. If for any given $n$ we have $\lambda_{n}^{+}\left(\sqrt{V_{1}} A \sqrt{V_{1}}\right)>0$, then

$$
\lambda_{n}^{+}\left(\sqrt{V_{2}} A \sqrt{V_{2}}\right) \geq \lambda_{n}^{+}\left(\sqrt{V_{1}} A \sqrt{V_{1}}\right) .
$$

Similarly, if for any given $n$ we have $\lambda_{n}^{-}\left(\sqrt{V_{1}} A \sqrt{V_{1}}\right)<0$, then

$$
\lambda_{n}^{-}\left(\sqrt{V_{2}} A \sqrt{V_{2}}\right) \leq \lambda_{n}^{-}\left(\sqrt{V_{1}} A \sqrt{V_{1}}\right) .
$$


Proof. Let us first assume that 0 is not an eigenvalue of $V_{1}$, so $V_{1}^{-1} \geq V_{2}^{-1} \geq 0$ are self-adjoint operators.

Now suppose that $\lambda_{n}^{-}\left(\sqrt{V_{1}} A \sqrt{V_{1}}\right)<0$ and set $\mathcal{S}_{-}=\{\psi:\langle\psi, A \psi\rangle \leq 0\}$. Using Theorem A.1 we may write

$$
\begin{aligned}
\lambda_{n}^{-}\left(\sqrt{V_{1}} A \sqrt{V_{1}}\right) & =\inf _{L: \operatorname{dim} L=n}\left[\sup _{\psi \in L} \frac{\left\langle\psi, \sqrt{V_{1}} A \sqrt{V_{1}} \psi\right\rangle}{\|\psi\|^{2}}\right] \\
& =\inf _{L \subset \mathcal{D}\left(V_{1}^{-1}\right): \operatorname{dim} L=n}\left[\sup _{\psi \in L} \frac{\langle\psi, A \psi\rangle}{\left\langle\psi, V_{1}^{-1} \psi\right\rangle}\right] \\
& =\inf _{L \subset \mathcal{D}\left(V_{1}^{-1}\right): \operatorname{dim} L=n, L \subset \mathcal{S}_{-}}\left[\sup _{\psi \in L} \frac{\langle\psi, A \psi\rangle}{\left\langle\psi, V_{1}^{-1} \psi\right\rangle}\right] .
\end{aligned}
$$

In particular, there exists an $n$-dimensional subspace $L_{n} \subset \mathcal{D}\left(V_{1}^{-1}\right)$ such that $L_{n} \subset$ $\mathcal{S}_{-}$. Using Theorem A.1 again we get

$$
\lambda_{n}^{-}\left(\sqrt{V_{2}} A \sqrt{V_{2}}\right)=\inf _{L \subset \mathcal{D}\left(V_{2}^{-1}\right): \operatorname{dim} L=n,}\left[\sup _{\psi \in L} \frac{\langle\psi, A \psi\rangle}{\left\langle\psi, V_{2}^{-1} \psi\right\rangle}\right] .
$$

Since for $L=L_{n}$ clearly

$$
\sup _{\psi \in L_{n}} \frac{\langle\psi, A \psi\rangle}{\left\langle\psi, V_{2}^{-1} \psi\right\rangle}<0
$$

we have $\lambda_{n}^{-}\left(\sqrt{V_{2}} A \sqrt{V_{2}}\right)<0$, which together with (A.126) implies

$$
\lambda_{n}^{-}\left(\sqrt{V_{2}} A \sqrt{V_{2}}\right)=\inf _{L \subset \mathcal{D}\left(V_{2}^{-1}\right): \operatorname{dim} L=n, L \subset \mathcal{S}_{-}}\left[\sup _{\psi \in L} \frac{\langle\psi, A \psi\rangle}{\left\langle\psi, V_{2}^{-1} \psi\right\rangle}\right] .
$$

Taking now into account the fact that for any $L \subset \mathcal{S}_{-}$and $\psi \in L$, we have $\langle\psi, A \psi\rangle \leq$ 0 , and the inequality $V_{1}^{-1} \geq V_{2}^{-1}$, we deduce from (A.125) and (A.127) the following inequality:

$$
\begin{aligned}
& \lambda_{n}^{-}\left(\sqrt{V_{2}} A \sqrt{V_{2}}\right) \\
(\mathrm{A} .128) & =\inf _{L \subset \mathcal{D}\left(V_{2}^{-1}\right): \operatorname{dim} L=n, L \subset \mathcal{S}_{-}}\left[\sup _{\psi \in L} \frac{\langle\psi, A \psi\rangle}{\left\langle\psi, V_{2}^{-1} \psi\right\rangle}\right] \\
& \leq \inf _{L \subset \mathcal{D}\left(V_{1}^{-1}\right): \operatorname{dim} L=n, L \subset \mathcal{S}_{-}}\left[\sup _{\psi \in L} \frac{\langle\psi, A \psi\rangle}{\left\langle\psi, V_{1}^{-1} \psi\right\rangle}\right]=\lambda_{n}^{-}\left(\sqrt{V_{1}} A \sqrt{V_{1}}\right),
\end{aligned}
$$

which implies the inequality (A.124) under the additional assumption that 0 is not an eigenvalue of $V_{1}$. To get rid of it let us take any positive compact operator $V_{0}$, with 0 not an eigenvalue of $V_{0}$, and $\delta>0$. Note that $\lambda_{n}^{-}(\sqrt{V} A \sqrt{V})$ is a continuous function of $V$ in the operator norm; hence, if $\lambda_{n}^{-}\left(\sqrt{V_{1}} A \sqrt{V_{1}}\right)<0$, for sufficiently small $\delta>0$, we must have $\lambda_{n}^{-}\left(\sqrt{V_{1}+\delta V_{0}} A \sqrt{V_{1}+\delta V_{0}}\right)<0$, so it follows from (A.128) that

$$
\lambda_{n}^{-}\left(\sqrt{V_{2}+\delta V_{0}} A \sqrt{V_{2}+\delta V_{0}}\right) \leq \lambda_{n}^{-}\left(\sqrt{V_{1}+\delta V_{0}} A \sqrt{V_{1}+\delta V_{0}}\right) .
$$


Sending $\delta$ to zero in (A.129), we arrive at (A.124) without additional conditions.

The inequality (A.123) is proved in a similar way.

Appendix B. An "a priori" estimate for the exponential decay of resolvents. The following result is proven in [FK3, Lemmas 12 and 13] and [FK5, Lemmas 15 and 16]. We use $\nabla^{\sharp}$ to denote $\nabla$ if $W=A$ and $\nabla^{\times}$if $W=M$. Recall $\chi_{x}$ denotes the characteristic function of a cube of side 1 centered at $x$.

Proposition B.1. Let $\varepsilon(x)$ be as in (4). Then there exists a finite constant $\alpha_{d}$, depending only on the dimension $d$, such that

$$
\left\|\chi_{x} \widetilde{R}(-1) \chi_{y}\right\| \leq \alpha_{d} e^{-\beta|x-y|}
$$

and

$$
\left\|\chi_{x} \nabla^{\sharp} \widetilde{R}(-1) \chi_{y}\right\| \leq \alpha_{d} \sqrt{\varepsilon_{+}}\left(\sqrt{\varepsilon_{-}}+\frac{1}{\sqrt{\varepsilon_{-}}}\right) e^{-\beta|x-y|},
$$

for all $x, y \in \mathbb{R}^{d}$, where

$$
\beta=\frac{1}{4\left[\varepsilon_{-}^{-1}+2\right]}
$$

Appendix C. Some operator estimates. Let $\chi_{x}$ be the characteristic function of the unit cube centered at $x$. For any $\varphi \in L^{2}\left(\mathbb{R}^{d} ; \mathbb{C}^{\nu}\right)$, we consider the decomposition

$$
\varphi=\sum_{m \in \mathbb{Z}^{d}} \varphi_{m}, \varphi_{m}=\chi_{m} \varphi
$$

If $T$ is a bounded operator in $L^{2}\left(\mathbb{R}^{d} ; \mathbb{C}^{\nu}\right)$, we will consider the following decomposition of $T$ associated with (C.133):

$$
T=\sum_{n, m \in \mathbb{Z}^{d}} T_{n m}, T_{n m}=\chi_{n} T \chi_{m}
$$

It is convenient to introduce the following normed space of operators:

$$
\mathcal{F}=\left\{T ;\|T\|_{\mathcal{F}} \equiv\left(\sum_{n, m \in \mathbb{Z}^{d}}\left\|T_{n m}\right\|^{2}\right)^{1 / 2}<\infty\right\} .
$$

Lemma C.1. $\mathcal{F}$ is a Banach space. Moreover, for $T, S \in \mathcal{F}$ we have

$$
\begin{array}{r}
\|T\| \leq\|T\|_{\mathcal{F}}, \\
\|S T\|_{\mathcal{F}} \leq\|S\|_{\mathcal{F}}\|T\|_{\mathcal{F}}, \\
\|T\|_{\mathcal{F}}=\left\|T^{*}\right\|_{\mathcal{F}},
\end{array}
$$

and

$$
T^{*} T \leq\|T\|_{\mathcal{F}}^{2} I .
$$

Proof. It is routine to verify that $\mathcal{F}$ is a Banach space. 
The inequality (C.136) follows from

$$
\begin{aligned}
\|T \varphi\|^{2} & =\sum_{n \in \mathbb{Z}^{d}}\left\|\sum_{m \in \mathbb{Z}^{d}} T_{n m} \varphi_{m}\right\|^{2} \\
& \leq \sum_{n \in \mathbb{Z}^{d}}\left(\sum_{m \in \mathbb{Z}^{d}}\left\|T_{n m}\right\|^{2} \sum_{m \in \mathbb{Z}^{d}}\left\|\varphi_{m}\right\|^{2}\right)=\|T\|_{\mathcal{F}}^{2}\|\varphi\|^{2} .
\end{aligned}
$$

To prove (C.137), note

$$
\begin{aligned}
\|S T\|_{\mathcal{F}}^{2} & =\sum_{n, m \in \mathbb{Z}^{d}}\left\|(S T)_{n m}\right\|^{2} \\
& =\sum_{n, m \in \mathbb{Z}^{d}}\left\|\sum_{u \in \mathbb{Z}^{d}} S_{n u} T_{u m}\right\|^{2} \leq \sum_{n, m \in \mathbb{Z}^{d}}\left(\sum_{u \in \mathbb{Z}^{d}}\left\|S_{n u}\right\|\left\|T_{u m}\right\|\right)^{2} \\
& \leq \sum_{n, m \in \mathbb{Z}^{d}}\left(\sum_{u \in \mathbb{Z}^{d}}\left\|S_{n u}\right\|^{2}\right)\left(\sum_{u \in \mathbb{Z}^{d}}\left\|T_{u m}\right\|^{2}\right)=\|S\|_{\mathcal{F}}^{2}\|T\|_{\mathcal{F}}^{2}
\end{aligned}
$$

The equality (C.138) follows from the definition of $\|T\|_{\mathcal{F}}$. The operator inequality (C.139) follows from $T^{*} T \leq\|T\|^{2} I$ and (C.136).

The following is an immediate consequence of Lemma C.1.

Corollary C.2. Let $\Gamma$ be the positive self-adjoint operator on $L^{2}\left(\mathbb{R}^{d} ; \mathbb{C}^{\nu}\right)$, given by multiplication by the function

$$
\gamma(x)=\sum_{m \in \mathbb{Z}^{d}} \gamma_{m} \chi_{m}(x),
$$

where $\left\{\gamma_{m} ; m \in \mathbb{Z}^{d}\right\}$ is a given sequence of strictly positive numbers, and let $T$ be a bounded operator in $L^{2}\left(\mathbb{R}^{d} ; \mathbb{C}^{\nu}\right)$. Then if $T \Gamma \in \mathcal{F}$, i.e.

$$
\|T \Gamma\|_{\mathcal{F}}^{2}=\sum_{n, m \in \mathbb{Z}^{d}}\left\|T_{n m}\right\|^{2} \gamma_{m}^{2}<\infty
$$

we have the following operator inequality:

$$
T^{*} T \leq\|T \Gamma\|_{\mathcal{F}}^{2} \Gamma^{-2} .
$$

Proof. We have

$$
\begin{aligned}
T^{*} T & =\Gamma^{-1} \Gamma T^{*} T \Gamma \Gamma^{-1} \leq\left\|\Gamma T^{*} T \Gamma\right\| \Gamma^{-2} \\
& =\|T \Gamma\|^{2} \Gamma^{-2} \leq\|T \Gamma\|_{\mathcal{F}}^{2} \Gamma^{-2} .
\end{aligned}
$$

Appendix D. An estimate on traces. We use the following lemma.

Lemma D.1. Let $R$ be a bounded self-adjoint operator, and let $P$ and $Q$ be two orthogonal projections. Then

$$
\operatorname{Tr}(P R Q R P) \leq 4\left\{\operatorname{Tr}\left[(P|R| P)^{2}\right]\right\}^{1 / 2}\left\{\operatorname{Tr}\left[(Q|R| Q)^{2}\right]\right\}^{1 / 2}
$$


Proof. We write

$$
R=R_{+}-R_{-}, \text {with } R_{ \pm} \geq 0, R_{+} R_{-}=R_{-} R_{+}=0, \text { so }|R|=R_{+}+R_{-} .
$$

Thus

$$
\begin{aligned}
\operatorname{Tr}(P R Q R P) & =\|P R Q\|_{2}^{2} \\
& \leq 2\left(\left\|P R_{+} Q\right\|_{2}^{2}+\left\|P R_{-} Q\right\|_{2}^{2}\right) .
\end{aligned}
$$

Without loss of generality, we may assume that $P|R| P$ and $Q|R| Q$ are HilbertSchmidt operators; it follows that $P R_{ \pm} P$ and $Q R_{ \pm} Q$ are also Hilbert-Schmidt, so $R_{ \pm}^{1 / 2} P, R_{ \pm}^{1 / 2} Q \in \mathcal{T}_{4}$. It follows that

$$
\begin{aligned}
\left\|P R_{ \pm} Q\right\|_{2}^{2} & =\operatorname{Tr}\left(P R_{ \pm} Q R_{ \pm} P\right)=\operatorname{Tr}\left(R_{ \pm}^{1 / 2} P R_{ \pm} Q R_{ \pm}^{1 / 2}\right) \\
& \leq\left\|R_{ \pm}^{1 / 2} P R_{ \pm}^{1 / 2}\right\|_{2}\left\|R_{ \pm}^{1 / 2} Q R_{ \pm}^{1 / 2}\right\|_{2}=\left\|P R_{ \pm} P\right\|_{2}\left\|Q R_{ \pm} Q\right\|_{2}
\end{aligned}
$$

Furthermore,

$$
\begin{aligned}
\left\|P R_{ \pm} P\right\|_{2}^{2} & =\operatorname{Tr}\left(P R_{ \pm} P R_{ \pm} P\right)=\operatorname{Tr}\left(R_{ \pm}^{1 / 2} P R_{ \pm} P R_{ \pm}^{1 / 2}\right) \\
& \leq \operatorname{Tr}\left(R_{ \pm}^{1 / 2} P\left(R_{+}+R_{-}\right) P R_{ \pm}^{1 / 2}\right) \\
& =\operatorname{Tr}\left[\left(R_{+}+R_{-}\right)^{1 / 2} P R_{ \pm} P\left(R_{+}+R_{-}\right)^{1 / 2}\right] \\
& \leq \operatorname{Tr}\left[\left(R_{+}+R_{-}\right)^{1 / 2} P\left(R_{+}+R_{-}\right) P\left(R_{+}+R_{-}\right)^{1 / 2}\right] \\
& =\operatorname{Tr}\left[P\left(R_{+}+R_{-}\right) P\left(R_{+}+R_{-}\right) P\right]=\operatorname{Tr}\left[(P|R| P)^{2}\right]
\end{aligned}
$$

with an analogous estimate for $\left\|Q R_{ \pm} Q\right\|_{2}^{2}$. The estimate (D.149) now follows from (D.151), (D.152), and (D.153).

\section{REFERENCES}

[AADh] S. Alama, M. Avellaneda, P. Deift, and R. Hampel, On the existence of eigenvalues of a divergence form operator $A+\lambda B$ in a gap of $\sigma(A)$, Asymptotic Anal., 8 (1994), pp. 311-344.

[ADH] S. Alama, P. Deift, and R. Hampel, Eigenvalues branches of the Schrödinger operator $H-\lambda W$ in a gap of $\sigma(H)$, Comm. Math. Phys., 121 (1989), pp. 291-321.

[An] P. W. Anderson, A question of classical localization. A theory of white paint, Philosoph. Magazine, B 53 (1958), pp. 505-509.

[BS] M. Sh. Birman And M. Z. SOlomyak, $L^{2}$-theory of the Maxwell operator in arbitrary domains, Russian Math. Surveys, 42 (1987), pp. 75-96.

[BL] S. I. Boyarchenko And S. Z. Levendorskit, An Asymptotic Formula for the Number of Eigenvalues Branches of a Divergence Form Operator $A+\lambda B$ in a Spectral Gap of $A$, Rostov Institute of National Economy, Russia, 1997, preprint.

[DE] Development and applications of materials exhibiting photonic band gaps, J. Opt. Soc. Amer. B, 10 (1993), pp. 280-413.

[FK1] A. Figotin AND A. KLEIN, Localization phenomenon in gaps of the spectrum of random lattice operators, J. Statist. Phys., 75 (1994), pp. 997-1021.

[FK2] A. Figotin AND A. KLein, Localization of electromagnetic and acoustic waves in random media. Lattice model, J. Statist. Phys., 76 (1994), pp. 985-1003.

[FK3] A. Figotin And A. Klein, Localization of classical waves I: Acoustic waves, Comm. Math. Phys., 180 (1996), pp. 439-482. 
[FK4] A. Figotin And A. Klein, Localized classical waves created by defects, J. Statist. Phys., 86 (1997), pp. $165-177$.

[FK5] A. Figotin AND A. KLein, Localization of classical waves II: Electromagnetic waves, Comm. Math. Phys., 184 (1997), pp. 411-441.

[FKu1] A. Figotin And P. Kuchment, Band-gap structure of spectra of periodic dielectric and acoustic media. I. Scalar model, SIAM J. Appl. Math., 56 (1996), pp. 68-88.

[FKu2] A. Figotin And P. Kuchment, Band-gap structure of spectra of periodic dielectric and acoustic media. II. $2 D$ photonic crystals, SIAM J. Appl. Math., 56 (1996), pp. 15611620.

[HJ] P. M. Hui And N. F. Johnson, Photonic band-gap materials, in Solid State Physics, Vol. 49, H. Ehrenreich and F. Spaepen, eds., Academic Press, New York, 1995, pp. 151-203.

[JMW] J. Joannopoulos, R. Meade, and J. Winn, Photonic Crystals, Princeton University Press, Princeton, NJ, 1995.

[J1] S. John, Localization of light, Phys. Today, May 1991.

[J2] S. John, The Localization of light, in Photonic Band Gaps and Localization, NATO Adv. Sci. Inst. Ser. B Phys., 308, 1993.

[Ka] T. Kato, Perturbation Theory for Linear Operators, Springer-Verlag, Berlin, New York, 1976.

[Kl] M. KLAuS, Some aplications of the Birman-Schwinger pinciple, Helv. Phys. Acta, 55 (1982), pp. 49-68.

[Kp] F. KLOPP, Internal Lifshits Tails for Random Perturbations of Periodic Schrödinger Operators, Université Paris Nord, France, 1996, preprint.

[Ku] P. Kuchment, Floquet Theory for Partial Differential Equations, Birkhäuser-Verlag, Basel, 1993.

[M] J. Maynard, Acoustic Anderson localization, in Random Media and Composites, SIAM, Philadelphia, PA, 1988.

[RS1] M. Reed ANd B. Simon, Methods of modern mathematical physics, Vol. I, Functional Analysis, Academic Press, New York, 1980.

[RS4] M. REed And B. Simon, Methods of modern mathematical physics, Vol. IV, Analysis of Operators, Academic Press, New York, 1978.

[S] L. Schiff, Quantum Mechanics, McGraw-Hill, New York, 1968.

[Sc] Scattering and Localization of Classical Waves, P. Sheng, ed., World Scientific, Rivers Edge, NJ, 1990.

[VP] P. R. Villeneuve And M. Piché, Photonic band gaps in periodic dielectric structures, Progr. Quant. Electr., 18 (1994), pp. 153-200. 